\title{
Talklüfte im Zentralen Aaregranit der Schöllenen-Schlucht (Kanton Uri, Schweiz)
}

\section{Journal Article}

Author(s):

Bucher, Stefan P.; Löw, Simon

Publication date:

2009

Permanent link:

https://doi.org/10.3929/ethz-b-000021672

Rights / license:

In Copyright - Non-Commercial Use Permitted

Originally published in:

Swiss Journal of Geosciences 102(3), https://doi.org/10.1007/s00015-009-1334-0 


\title{
Talklüfte im Zentralen Aaregranit der Schöllenen-Schlucht (Kanton Uri, Schweiz) Unloading joints in the central Aar granite of the Schöllenen Gorge (canton Uri, Switzerland)
}

\author{
STEFAn P. Bucher ${ }^{1,2} \&$ Simon LOEW ${ }^{2}$ \\ Key words: granite, Aar massif, fractographic markings, unloading fractures, exfoliation, jointing, rockfall hazards
}

\section{ZUSAMMENFASSUNG}

Talklüfte können im Zusammenhang mit Strasseneinschnitten, Wasserkraftanlagen und geologischen Massenbewegungen in steilen Talflanken eine wichtige Rolle spielen. Die Schöllenenschlucht im Reusstal (zentrale Schweizer Alpen) bildet ein ideales Untersuchungsgebiet für das Studium der Eigenschaften und Entstehungsmechanismen solcher Talklüfte. Die SchöllenenSchlucht liegt am Südrand des Zentralen Aaregranits, welcher vor rund 300 Mio. Jahren intrudierte, während der Alpenbildung nur lokal duktil deformiert und anschliessend an die Oberfläche gehoben wurde. Die bis zu $600 \mathrm{~m}$ tiefe Schlucht bietet einen idealen Einblick in das komplexe Trennflächengefüge des Plutons. Einen Einblick in das Berginnere erlauben zudem die Stollen des Kraftwerks Göschenen.

Die Kartierungsarbeit führte zur Unterscheidung von zwei vermutlich alpin-syntektonischen Kluftfamilien (S- und Q-Klüfte) sowie drei Generationen von Entlastungsklüften (subhorizontale Dehnungsklüfte (L-Klüfte) und zwei Generationen von mittelsteilen bis steilen Talklüften $\left(T_{1}-\right.$ und $T_{2}-$ Klüfte)). Die zahlreichen S-Klüfte verlaufen meistens parallel zur alpinen Schieferung, d.h. ungefähr E-W streichend und steil gegen Süden einfallend. Die weniger häufigen Q-Klüfte fallen steil gegen SW ein, der Winkel zwischen den beiden Kluftfamilien beträgt 60 bis $80 \mathrm{Grad}$. Die räumliche Lage der verschiedenen Entlastungsklüfte wurde primär in Querprofilen durch die Schöllenen erfasst. Die ersten Entlastungsklüfte verlaufen horizontal, sie bildeten sich unabhängig von der Topographie möglicherweise während einer spätalpinen miocänen Hebung des Aarmassivs. Eine erste Generation von Talklüften $\left(T_{1}\right)$ fällt mit 30-45 Grad beidseitig talwärts ein und wurde vermutlich während einer frühen pleistozänen Eintalung gebildet. Die jüngsten Talklüfte $\left(T_{2}\right)$ sind parallel zur heutigen Taloberfläche der Schöllenenschlucht orientiert, d.h. sie liegen parallel zu den mit spätpleistozänen Gletscherschliffen markierten Felsoberflächen. Die Frequenz sowie die Grösse dieser Klüfte nehmen zumindest in einem Stollen gegen das Berginnere ab und konnten bis rund 150 Meter ins Berginnere nachgewiesen werden.

Fraktographische Untersuchungen, d.h. die Untersuchung von Bruchstrukturen auf Kluftoberflächen (hauptsächlich Federmarken mit einer Grösse von 5 bis $10 \mathrm{~m}$ ) bestätigten diese relative Alterabfolge. Ebenso konnten dadurch jeweils der Ursprungspunkt einer Kluft und die Bruchfortpflanzungsrichtung bestimmt werden. Es zeigte sich dabei stets, dass grosse Talklüfte (bis über hundert Meter Länge) aus mehreren 5-10 Meter grossen Extensionsklüften bestehen, welche durch primär subhorizontale stabile Rissausbreitung entstanden sind. Grosse Talklüfte bilden sich bevorzugt in Graniten mit nur geringer alpin-tektonischer Überprägung.

Die Talklüfte der Schöllenen bilden sich nicht als Scherbrüche, wie dies von früheren Autoren angenommen wurde, sondern als Extensionsrisse in einem kompressiven Spannungsfeld. Das räumliche Auftreten und die beobachteten Grössen der Talklüfte können teilweise mit quantitativen
Bruchkriterien, wie sie für hohlraumnahe Extensionsrisse in Tunnelwerken entwickelt wurden, erklärt werden. Wesentlich für den Ort und die Grösse der Talklüfte sind neben den Deviatorspannungen und Zugspannungen auch die $\sigma_{3} / \sigma_{1}$ Hauptspannungsverhältnisse. Erste numerische Modellrechnungen zu einer mehrphasigen glazialen Eintalung vermögen die beobachteten Bildungsbereiche und Eigenschaften der Talklüfte grob abzubilden.

\section{EXTENDED ABSTRACT}

The Schöllenen Gorge in the Reuss Valley of the Central Swiss Alps (Figs. 1 and 2) is a famous tourist attraction and ideal location for the study of the properties and formation mechanisms of uplift and post-uplift unloading joints. The gorge is situated in the southern part of the Central Aar Granite, a granitic batholith which intruded about 300 million years ago. The magmatic fabric of this batholith (Fig. 4) has only been locally modified during Alpine tectonic and metamorphic overprinting, mainly in the vicinity of ductile-brittle shear zones. The up to $600 \mathrm{~m}$ deep gorge provides an ideal opportunity to study the complex fracture systems of the batholith, and tunnels of the Göschenen hydropower system allow the study of the fracture patterns below ground surface.

Outcrop, tunnel and remote mapping of fractures in the study area lead to the recognition of two probably syntectonic (Oligocene-Miocene) joint sets (S and $\mathrm{Q}$ joints) and three generations of uplift and post-uplift joints (unloading joints). The frequent $S$ joints run nearly parallel to the Alpine schistosity, i.e. striking approximately E-W and dipping steeply to the south (Figs. 5 and 7). The less frequent $\mathrm{Q}$ joints dip steeply to SW; the angle between the two joint sets ranges between 60 and 80 degrees. The first generation of uplift joints (called L- joints) is subhorizontal and probably related to Alpine extensional veins filled with fissure quartz (Zerrklüfte). These veins formed during the late Alpine (Miocene) uplift of the Aar Granite (Mullis 1996). A first generation of post-uplift joints ( $\mathrm{T}_{1}$ joints) strikes parallel to the valley axes and dip with $30-45$ degrees towards the valley bottom. This set probably formed during an earlier stage of glacial valley erosion in the Pleistocene (Figs. 9-11). The youngest generation of post-uplift joints ( $\mathrm{T}_{2}$ joints) is orientated parallel to the present ground surface of the Schöllenen Gorge and to erosional surfaces with glacial striations (Figs. 9-11 and 21). The frequency and size of these joints seems to decrease with depth below the ground surface. In one tunnel, postuplift joints could be observed within a horizontal and vertical distance from the ground surface of 150 and 80 meters. Post-uplift joints only form in granites with a primary fabric that has not been intensively overprinted by brittle or ductile Alpine tectonic deformations.

Fractographic investigations, i.e. investigations of crack propagation markers on joint surfaces, confirm this relative age of the fracture sets and give valuable insights into the formation mechanisms of post-uplift joints. Post-uplift joints show intense and 5-10 meter long plumose markings and only rarely arrest lines (Figs. 18a and 20). It can be shown that sets of post-

\footnotetext{
${ }^{1}$ zur Zeit: Höheweg 2, CH-6472 Erstfeld. E-mail: ste_bucher@btgeo.ch

${ }^{2}$ Geologisches Institut, ETH Zürich, CH-8092 Zürich. E-mail: simon.loew@erdw.ethz.ch
} 
uplift joints join at pre-existing (uplift and syntectonic) fractures to form large (50-100 m sized) curved exfoliation structures (Fig. 19). The growth direction of the post-uplift joints is mainly in subhorizontal directions (Figs. 19 and 20).

Fractographic markings, spatial and depth distributions as well as the relative size of post-uplift fractures are explained within the mechanical framework of uniaxial and biaxial compression tests on intact granite samples and samples with artificial flaws. Most of these experiments have been carried out in the framework of studies related to brittle failure (spalling and rockbursting) around deep mining drifts and tunnels in hard rock's (e.g. Hoek \& Bieniawski 1965, Read et al. 1998, Eberhardt et al. 1999). As suggested already by Holzhausen \& Johnson (1979), post-uplift fractures form as extension fractures in a compressive stress field with small confining stress. Laboratory tests carried out on artificial Griffith cracks suggest that the macroscopic fracture size is mainly controlled by the ratio of the smallest to the largest principal stress $\left(\sigma_{3} / \sigma_{1}\right)$, the so-called spalling limit. In steep slopes this ratio should increase with depth below ground surface (Fig. 24c), leading to smaller exfoliation fractures with increasing depth. The spatial occurrence of post-uplift fractures along the surface topography is a function of the deviatoric stress level (Fig. 24a) and/or the development of local tensile stresses (Fig. 24d). Preliminary numerical simulations of these failure criteria in a multistage glacial erosion model (Fig. 23) allow some of the observed patterns of post-uplift fracture distributions to be reproduced.

Post-uplift joints in steep glacial valleys play an important role in valley erosion and in connection with the risk of rock falls, the safety of traffic corridors, and the inflow of water to near-surface tunnels and hydropower caverns. The depth dependant sizes, frequencies and hydraulic conductivities of these fractures can be directly related to the occurrence and magnitudes of the corresponding hazards.

\section{Einleitung}

\section{Kluftgenese und Untersuchungsziele}

Im Zusammenhang mit der Talbildung, der Entstehung von Felsstürzen und der Stabilität von Untertagebauwerken in den Alpen spielen Entlastungsklüfte oft eine wichtige Rolle. So wurde beispielsweise die Gotthard Autobahn A2 im Sommer 2006 während eines Monats geschlossen, da diese durch Blocksturzniedergänge gefährdet war, deren Ursache unter anderem Talklüfte waren. Trotz dieser grossen Bedeutung wurden solche Klüfte im Alpenraum bisher kaum wissenschaftlich untersucht und ihre Entstehung nur pauschal bewertet.

Klüfte können als Sprödbrüche ohne signifikante Scherformung durch völlig verschiedene Prozesse entstehen. Ein magmatisches Gestein wie der Zentrale Aaregranit durchläuft eine komplexe geologische Geschichte, von der Intrusion tief in der Erdkruste bis zur Erosion an der Erdoberfläche. Das Gestein passt sich den kontinuierlich ändernden Druck- und Temperaturbedingungen durch duktile und spröde Verformung an. Die Genese von Klüften in magmatischen Gesteinen wurde insbesondere von Cloos (1921, 1925), Jahns (1943), Segall (z. B. Segall \& Pollard 1983), Holzhausen (1989), Bankwitz (z.B. Bankwitz \& Bankwitz 1995) und Bahat (1991, Bahat et al. 2005) untersucht. Basierend auf diesen Untersuchungen können die Hauptstadien der Kluftbildung in Graniten den drei wesentlichsten Prozessen, welche Granitkörper oft durchlaufen, zugeordnet werden und die Klüfte in folgende genetische Gruppen unterteilt werden (Bahat 1991): Abkühlungsklüfte, syntektonische Klüfte und Entlastungsklüfte. Das Hauptaugenmerk wird in dieser Arbeit auf die Entlastungsklüfte gelegt.

Die Genese syntektonischer Klüfte ist sehr vielfältig und komplex, da sie oft in Interaktionen mit duktilen Deformationsstrukturen (z.B. Zangerl et al. 2006), unter verschiedenen P-T-Bedingungen und durch verschiedene Fluid-gekoppelte Prozesse (z.B. Jebrak 1997) entstehen können. Nach Bahat (1991) werden bei einer spröden syntektonischen Deformation in Graniten oft subvertikale Klüfte angelegt. Mehrphasige tektonische Ereignisse oder veränderliche Spannungsfelder können jedoch auch zu geneigten syntektonischen Klüften und mehreren Kluftfamilien führen. Kluftabstände sind in der Regel gering und zeigen teilweise eine Abhängigkeit zu tektonischen Störzonen. Syntektonische Klüfte sind oft mit Mineralparagenesen der entsprechenden P-T-Bedingungen verfüllt.

Wird das Gestein durch tektonische oder isostatische Prozesse gehoben, nimmt der Überlagerungsdruck ab, weil das überlagernde Gestein durch Erosion abgetragen wird. Diese Entlastung führt in der oberen Kruste (typischerweise ab 10-15 km Tiefe, Sibson 1977) zu elastischen und spröden Ausgleichsbewegungen und das Gestein dehnt sich in Richtung Oberfläche aus. Dadurch werden in einem spröden Gestein wie Granit relativ junge Klüfte gebildet. Es gibt nach Bahat (1991) zwei verschiedene Typen und Generationen von Entlastungsklüften: uplift und post-uplift joints. Als uplift joints

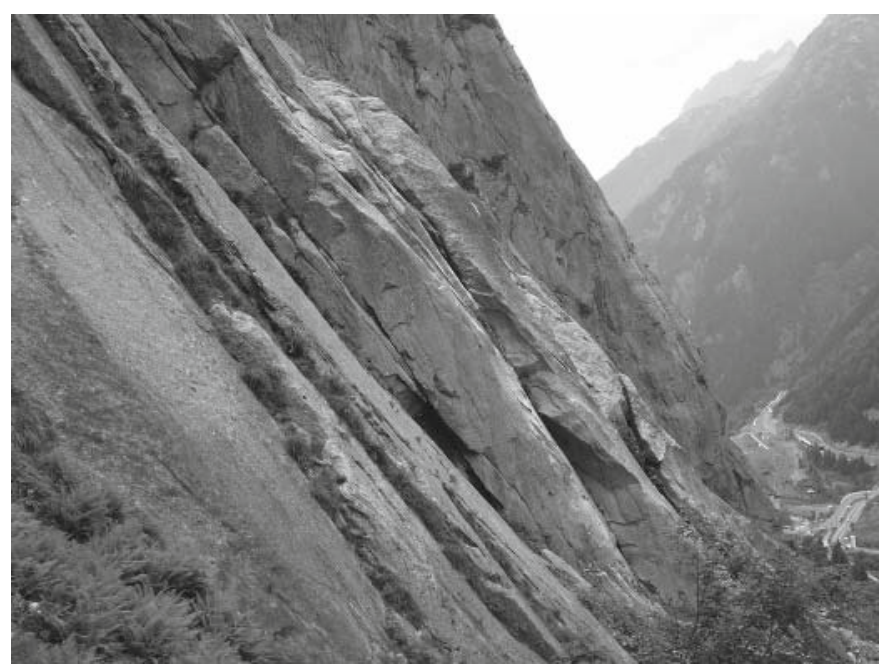

Fig. 1. Parallel zur Geländeoberfläche verlaufende, das Gestein in dm- bis mmächtige Platten durchtrennende Talklüfte auf der Westseite der Schöllenenschlucht (Blickrichtung N). 


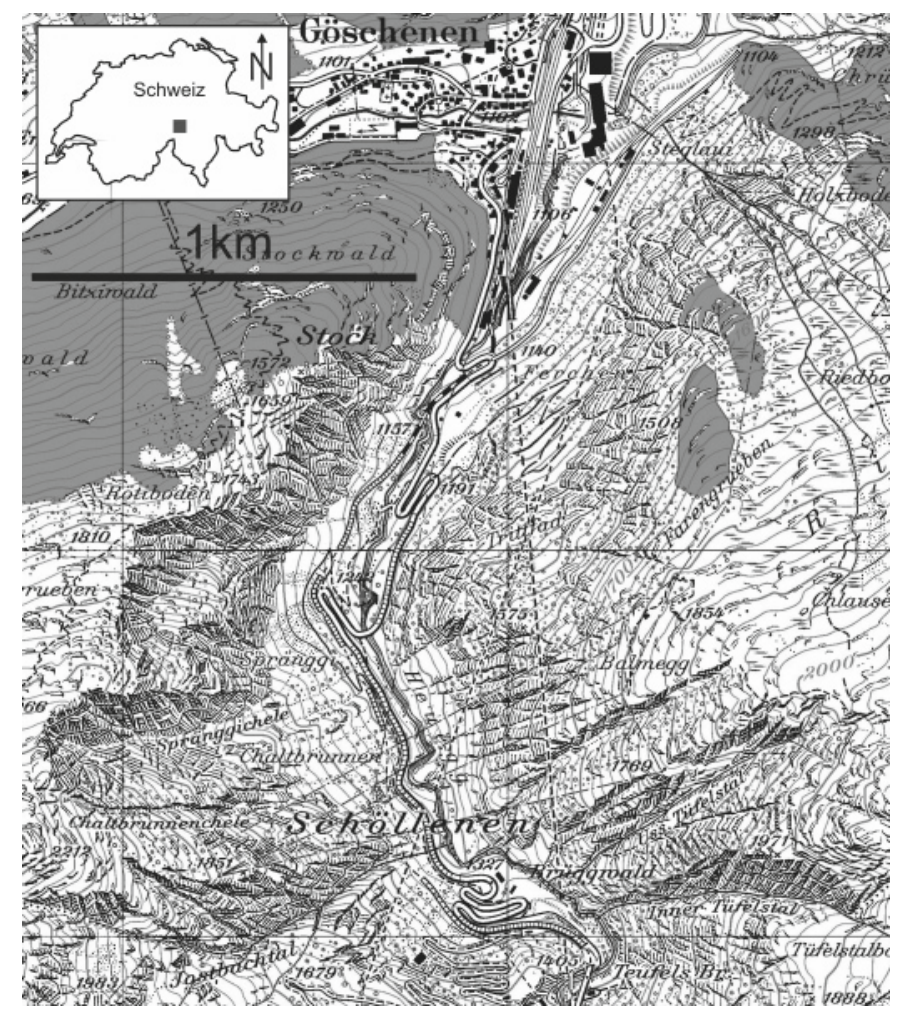

Fig. 2. Geographische Lage der Schöllenen im oberen Urner Reusstal.

oder Hebungsklüfte werden Entlastungsklüfte bezeichnet, die gebildet werden, noch bevor das Gestein die Oberfläche erreicht. Sie entwickeln sich unabhängig von der Topographie, diskordant zu magmatischen Fliesslinien, umfassen verschiedene Kluftfamilien und zeigen in der Regel grosse Kluftabstände.
Sobald der Pluton die Oberfläche erreicht, bildet sich ein neues Relief und post-uplift joints. Diese haben einen direkten geometrischen Bezug zur heutigen oder früheren Geländeoberfläche und können mit geomorphologischen Kriterien beschrieben werden. Post-uplift joints, welche das Gestein in parallele Platten spalten, werden auch als Bankungsklüfte (Bankwitz \& Bankwitz 1995) oder exfoliation joints (Bradley 1963; Bahat et al. 2005) bezeichnet. Oft werden diese Klüfte in Steinbrüchen beobachtet. Typisch ist der zunehmende Kluftabstand mit der Tiefe. Zu den post-uplift joints gehören auch die in dieser Arbeit behandelten Talklüfte. Von Talklüften spricht man vor allem im Zusammenhang mit talparallelen Klüften in steilen, oft glazial geprägten Tälern. Die Streichrichtung der Talklüfte folgt dem lokalen oder regionalen Talverlauf, ihre Fallazimute und Fallwinkel entsprechen oft dem topographischen Gefälle (Fig. 1).

In dieser Arbeit werden die Resultate einer Diplomarbeit (Bucher 2006) vorgestellt, welche die Eigenschaften und Genese der Klüfte, insbesondere der Talklüfte, in der berühmten, bis zu $600 \mathrm{~m}$ tiefen Schöllenenschlucht zwischen Göschenen und Andermatt zum Thema hatte (Fig. 2). Die hier vorgestellte Arbeit basiert auf umfangreichen direkten und indirekten Feldmessungen an Aufschlüssen und unter Tage (Tab. 1) sowie ersten numerischen Modellrechnungen.

\section{Geologische Verhältnisse im Untersuchungsgebiet}

Die Schöllenen liegt am Südrand des Zentralen Aaregranits, welcher das langgestreckte Rückgrat des Aar-Massivs bildet. Mit seinen $550 \mathrm{~km}^{2}$, einer Länge von über $100 \mathrm{~km}$ und einer maximalen Breite von 8-9 km im Raum Grimsel-Göscheneralp-Reusstal ist es der grösste Granitkörper der Schweiz (Fig. 3). Die Hauptintrusion erfolgte vor rund 300 Mio. Jahren während der variszischen Orogenese (Labhart 1999). Nach einer permischen Erosion und der Ablagerung der mesozo-

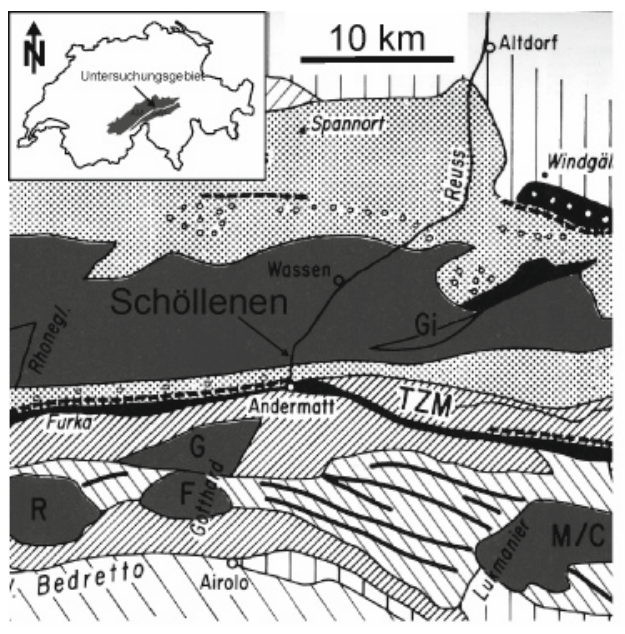

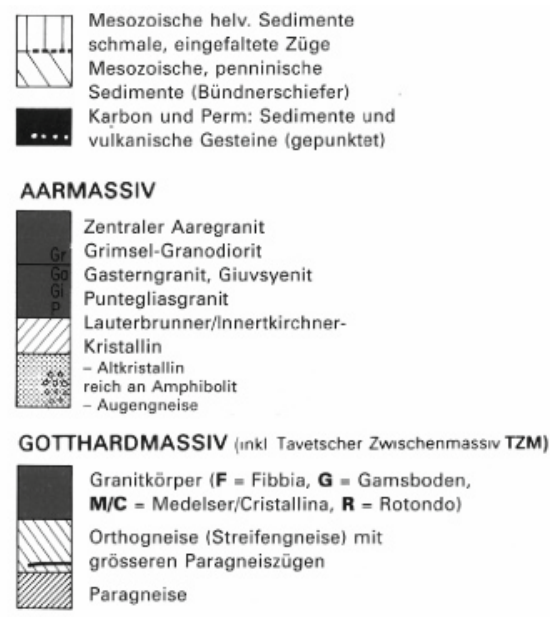

Fig. 3. Geologischer Überblick: Die Schöllenen am Südrand des Zentralen Aaregranits (Labhart 2001, leicht angepasst). 
Tabelle 1. Übersicht der Stollen, in welchen Kluftmessungen durchgeführt wurden (KWG $=$ Kraftwerk Göschenen).

\begin{tabular}{lll}
\hline Stollenbezeichnung & $\begin{array}{l}\text { Koordinaten } \\
\text { (Portal) }\end{array}$ & $\begin{array}{l}\text { Höhe } \\
{[\mathrm{m} \text { ü. M.] }}\end{array}$ \\
\hline Vorfluterstollen (KWG) & $687^{\prime} 713 / 169^{\prime} 108$ & 1089 \\
Kabelstollen (KWG) & $67^{\prime} 778 / 168^{\prime} 872$ & 1099 \\
Fensterstollen (KWG) & $687^{\prime} 245 / 168^{\prime} 657$ & 1388 \\
Armeestollen & $687^{\prime} 985 / 166^{\prime} 990$ & 1390 \\
\hline
\end{tabular}

ischen Sedimentabfolge gelangte das Aar-Massiv vor etwa 35 Mio. Jahren in den Bereich der alpinen Gebirgsbildung. Die Datierung der etwa 20 Mio. Jahre dauernden mehrphasigen alpinen Deformationen im Gebiet des Aar-Massivs basiert auf Analysen der Sedimentstrukturen am Nordrand des Massivs und ist bis heute umstritten (Milnes \& Pfiffner 1980; Burkhard 1999). Durch die Überlagerung der heute abgetragenen helvetischen, penninischen und ostalpinen Decken wurden die Gesteine des südlichen Aarmassivs schwach metamorph überprägt (Grünschieferfazies, ca. $400-450^{\circ} \mathrm{C}, 3-6$ kbar, Frey et al. 1980; Frey \& Ferreiro-Mählmann 1999; Challandes 2001). Der Peak der alpinen Metamorphose im zentralen Aar-Massiv wurde möglicherweise vor etwa 20 Mio. Jahren erreicht (Mullis 1996; Challandes 2001). Die alpinen Deformationen des Aar-Massivs beinhalten die Bildung duktiler mylonitischer Scherzonen (Marquer \& Gapais 1985; Challandes 2001; Laws 2001), einer alpinen Schieferung, welche den Zentralen Aaregranit selektiv erfasste, sowie spröder Verformungsstrukturen (Laws 2001).

Bisherige Untersuchungen über die Entstehung von Klüften im Aarmassiv und dem Zentralen Aaregranit sind sehr beschränkt: Liechti (1933) und Steck (1968) befassten sich vor allem mit den duktilen Verformungsstrukturen, Mullis (1996) mit den alpinen Zerrklüften, Eckardt (1957) sowie Laws (2001) untersuchten primär die Eigenschaften der grösseren sprödduktilen Scherzonen und nicht der Kluftsysteme. Wichtige Beschreibungen geometrischer Eigenschaften von Kluftfamilien finden sich vor allem in ingenieurgeologischen Projektdokumentationen (Keller et al. 1987; Kobel \& Partner AG 1999) und Diplomarbeiten (Frei 1999; Both 1999; Guldenfels 1999). Unweit der Schöllenen untersuchte Zangerl (2003) ausführlich die Sprödstrukturen des Gotthardmassivs.

Die Lithologie des Zentralen Aaregranits in der Schöllenen beschränkt sich grösstenteils auf mittel- bis grobkörnigen Granit. Der Granit besteht hauptsächlich aus Kalifeldspat, Plagioklas, Quarz und Biotit. Der Plagioklas wurde saussuritisiert und zeigt eine Füllung aus Serizit und Klinozoisit (Huber 1948). Der Biotit wurde teilweise zu Chlorit umgewandelt. Zusammen mit dem Auftreten von Epidot zeigt dies einen grünschieferfaziellen Metamorphosegrad an. Das Gefüge ist gleichkörnig bis porphyrisch. Der Granit in der Schöllenen ist zonenweise, d.h. im Bereich duktiler alpiner Scherung, stark geschiefert und vergneisst (Choukroune \& Gapais 1983). Die alpine Schieferung fällt meistens steil gegen Süden ein und streicht im Norden

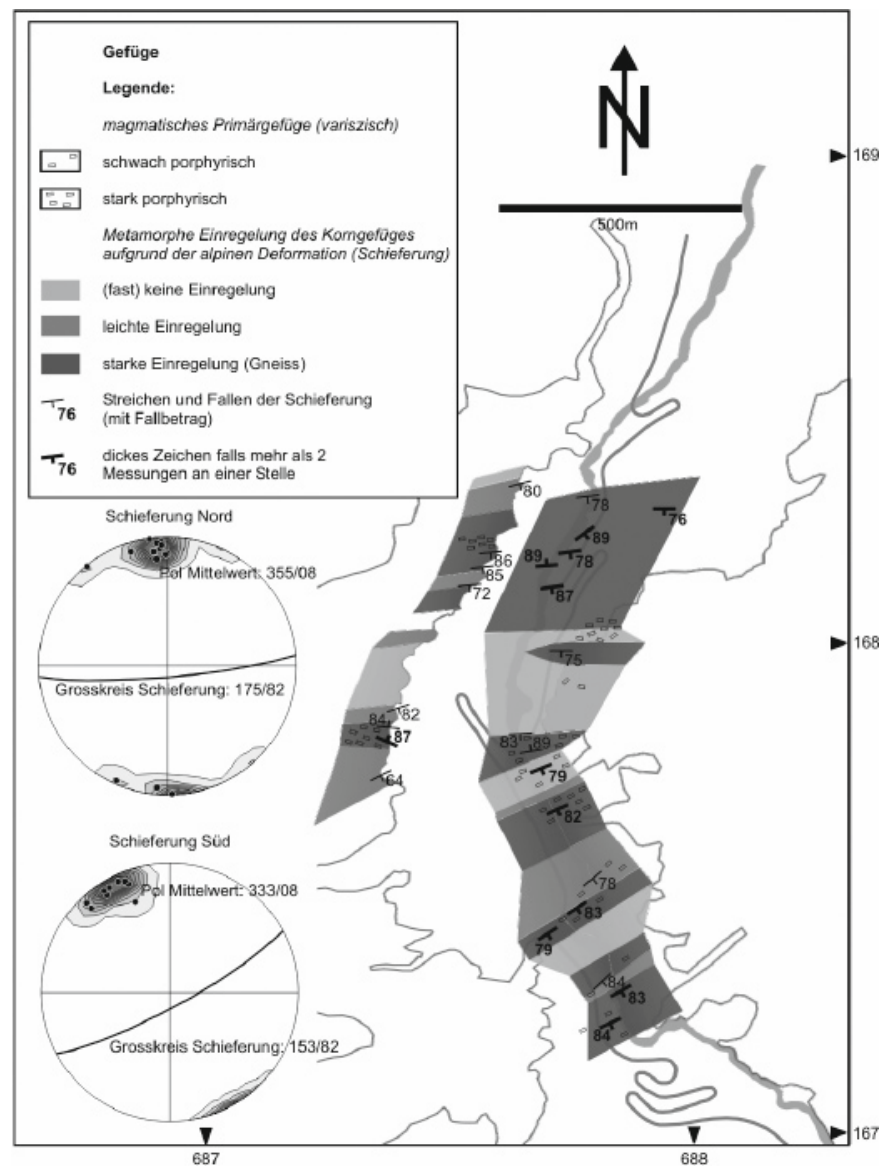

Fig. 4. Karte der Orientierung und Intensität der alpinen Schieferung im untersuchten Gebiet. Die in den Steroplots (untere Halbkugel, Schmidt'sches Netz, Kontur-Intervall im Abstand von zwei Prozent) dargestellten Pole entsprechen den Fallzeichen auf der Karte und wurden entsprechend der Anzahl Messungen gewichtet.

des Untersuchungsgebietes E-W und im Süden SW-NE. Das magmatische Gefüge, die metamorphe Einregelung des Korngefüges sowie die Raumstellung der alpinen Schieferung sind in Figur 4 dargestellt. Relativ häufig sind lamprophyrische und aplitische Ganggesteine. Die basischen Lamprophyre bestehen hauptsächlich aus Biotit und sind stark geschiefert (Huber 1948; Keller et al. 1987). Zahlreich sind basische Einschlüsse von maximal einigen Dezimetern Durchmesser, vorwiegend aus Plagioklas und Biotit. Diese sogenannten basischen Putzen werden als Ansammlung von Erstkristallisaten gedeutet (z.B. Labhart 1977).

\section{Kluftfamilien und ihre geometrischen Eigenschaften}

\section{Stereographische Analyse}

Um die Raumstellung und Verteilung der verschiedenen Kluftfamilien zu erfassen, wurde mittels Kompass die Orientierung 


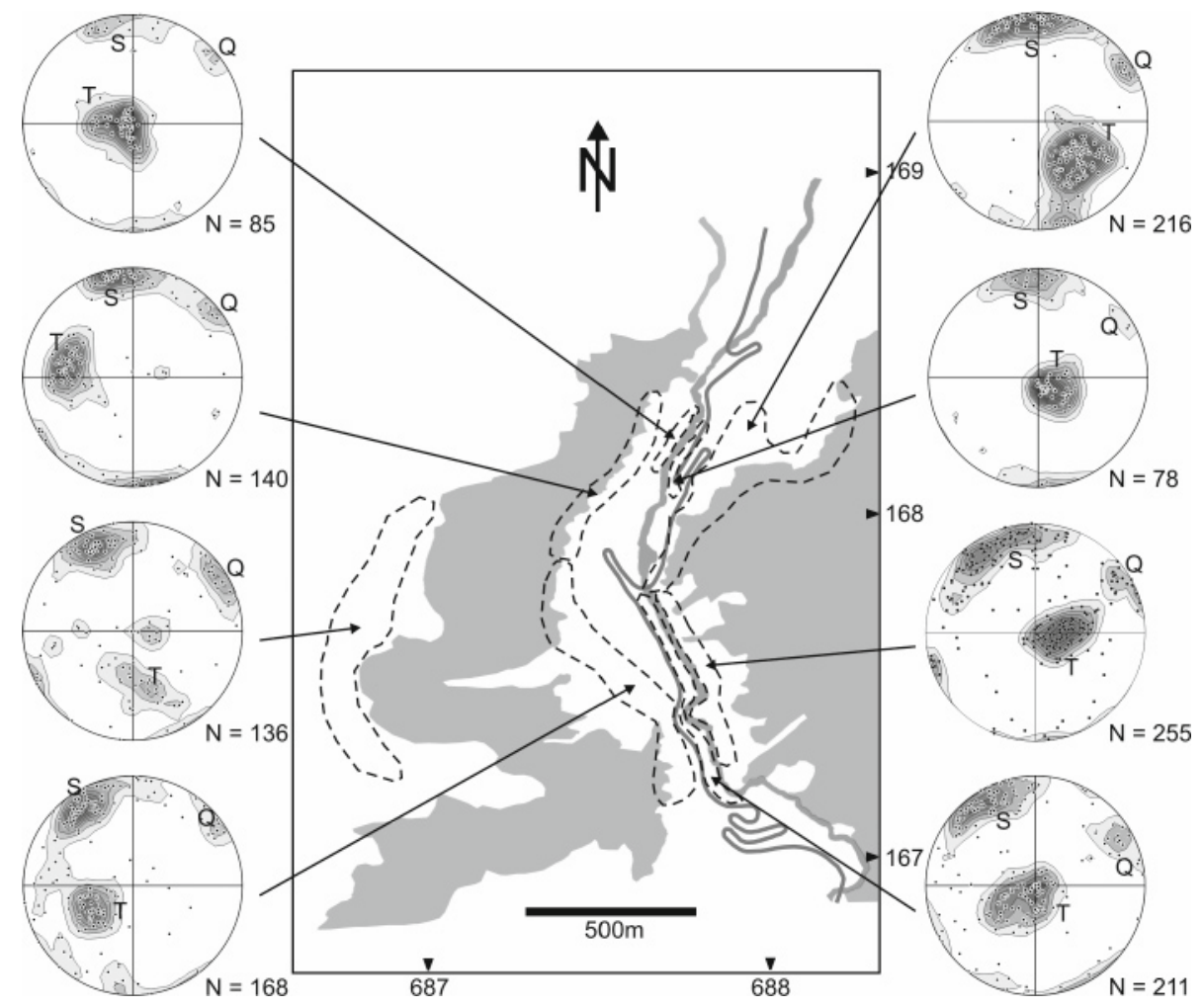

Fig. 5. Unterscheidung der S-, Q- und Talklüfte in Stereoplots (untere Halbkugel, Schmidt'sches Netz, Poldarstellung der Klüfte, Konturintervall im Abstand von zwei Prozent). Die hellgrauen Flächen in der Karte entsprechen den unzugänglichen steilen Felswänden der Schöllenenschlucht. von rund 1300 Klüften an der Oberfläche und mehr als 350 Klüften in den Stollen des Kraftwerks Göschenen eingemessen. Figur 5 zeigt die Raumstellung aller an der Oberfläche gemessenen Klüfte in Stereoplots (Schmidt'sches Netz, untere Halbkugel) für die ausgewählten Homogenbereiche (gestrichelt). Dies führt zu einer ersten Identifizierung von drei Kluftfamilien.

Als S-Klüfte werden in dieser Arbeit die ungefähr parallel zur Schieferung verlaufenden Klüfte bezeichnet. Sie fallen im nördlichen Teil des Untersuchungsgebiets steil SSE bis S ein (mittlere Orientierung Nord: 167/82), im südlichen Teil ist das Azimut analog zur Raumstellung der alpinen Schieferung rund $20 \mathrm{Grad}$ nach Osten rotiert (mittlere Orientierung Süd:147/76). S-Klüfte zeigen in der Regel relativ glatte Bruchoberflächen ohne neue Mineralausfällungen.

Weniger zahlreich sind steil SW einfallende Klüfte, die in dieser Arbeit als Q-Klüfte bezeichnet werden. Ihre Orientierung ist über das gesamte Untersuchungsgebiet konstant (mittlere Orientierung: 236/81). Im südlichen Teil verlaufen sie auffällig parallel zur Schöllenenschlucht. Q-Klüfte weisen oft einen rauhen Chloritbelag auf (Fig. 6). Sowohl S- wie Q-Klüfte finden sich in sehr ähnlicher Ausprägung im gesamten Gotthard-Gebiet und wurden von Zangerl et al. (2006) als alpinsyntektonische Klüfte eines länger andauernden Bruchprozesses interpretiert.

Die Identifikation der weiteren Kluftfamilien im Stereoplot (,T-Klüfte“) ist schwieriger, weil sie sich in der Raumstel- lung zum Teil überschneiden und ihre Orientierung in Abhängigkeit der lokalen Topographie verändern. Zum grössten Teil handelt es sich um Talklüfte, welche parallel zur Topographie orientiert sind und deshalb in jedem Stereoplot eine andere

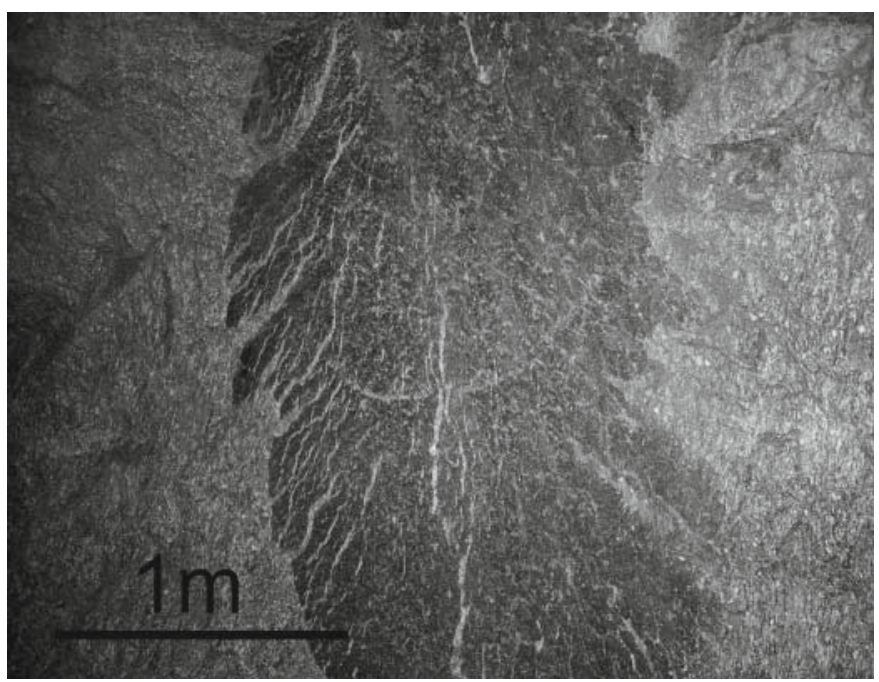

Fig. 6. Chloritbelegte, insbesondere im Randbereich sehr rauhe, getreppte QKluftfläche (aufgenommen im Fensterstollen des KW Göschenen). 

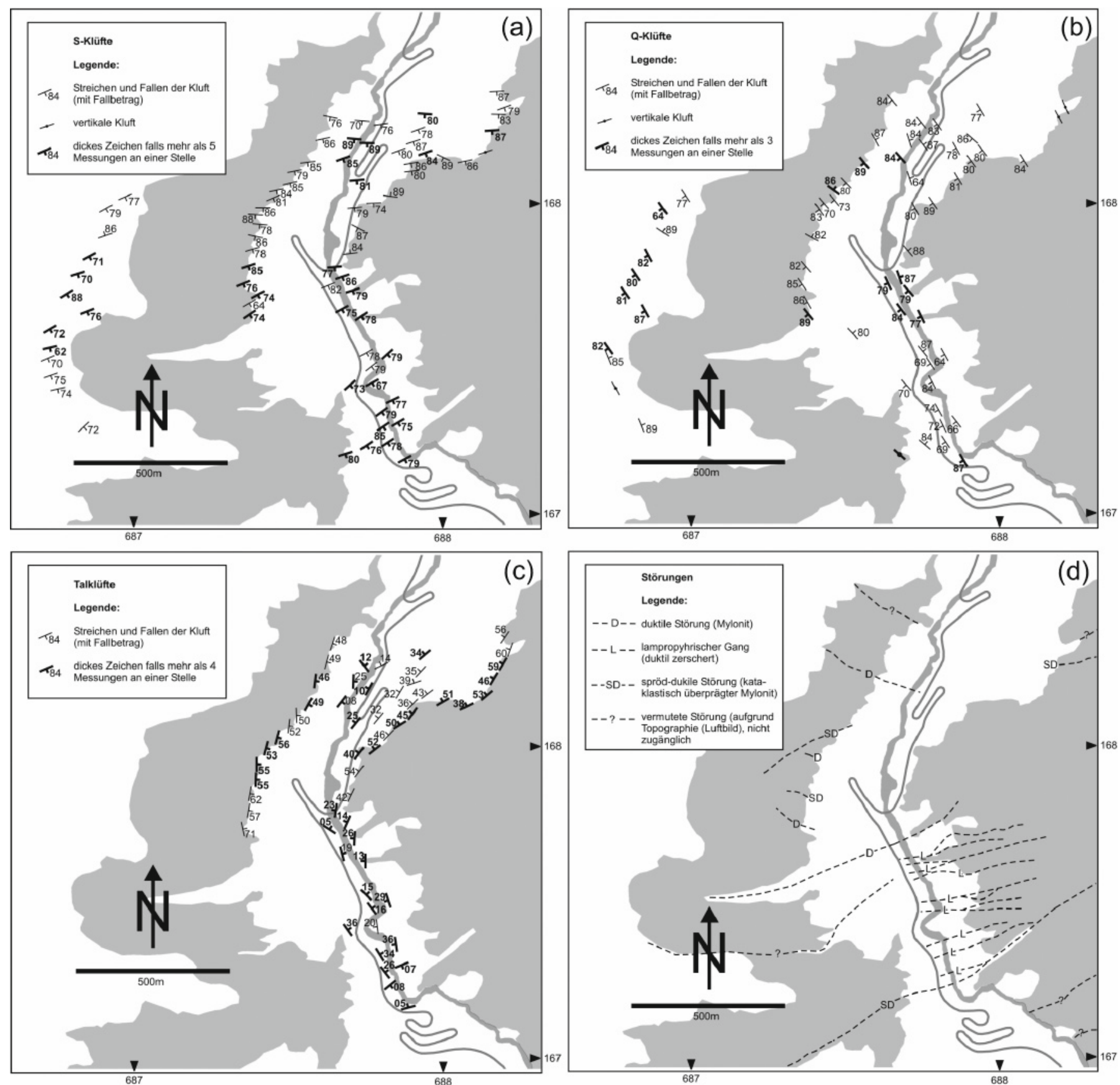

Fig. 7. Karten der drei mittels Stereoplots (vgl. Fig. 5) unterschiedenen Kluftfamilien: (a) steil S bis SE fallende S-Klüfte; (b) steil SW/(NE) fallende Q-Klüfte; (c) vorwiegend parallel zur Topographie orientierte, auf beiden Talseiten taleinwärts und gegen den Talboden flacher einfallende Klüfte (Talklüfte); (d) kartierte und mittels Luftbild interpolierte, spröde und spröd-duktile Störungen sowie zerscherte, lamprophyrische Gänge.

Lage aufweisen. Eine Differenzierung der verschiedenen Sets von Klüften mit mittlerem bis flachen Einfallen erfolgt in den nachfolgenden Kapiteln aufgrund detaillierter Aufschlussbeobachtungen.

Um die Abhängigkeit der Kluftraumstellung von der lokalen topographischen Situation zu erfassen, wurden für die drei oben genannten Kluftfamilien, die sogenannten S-, Q- und „T“-Klüfte, jeweils eine separate Karte erstellt (Fig. 7a-c). An jedem Aufschluss wurden wenn möglich mehrere Messungen durchgeführt und deren Mittelwert als dickes Fallzeichen auf den Karten dargestellt. 


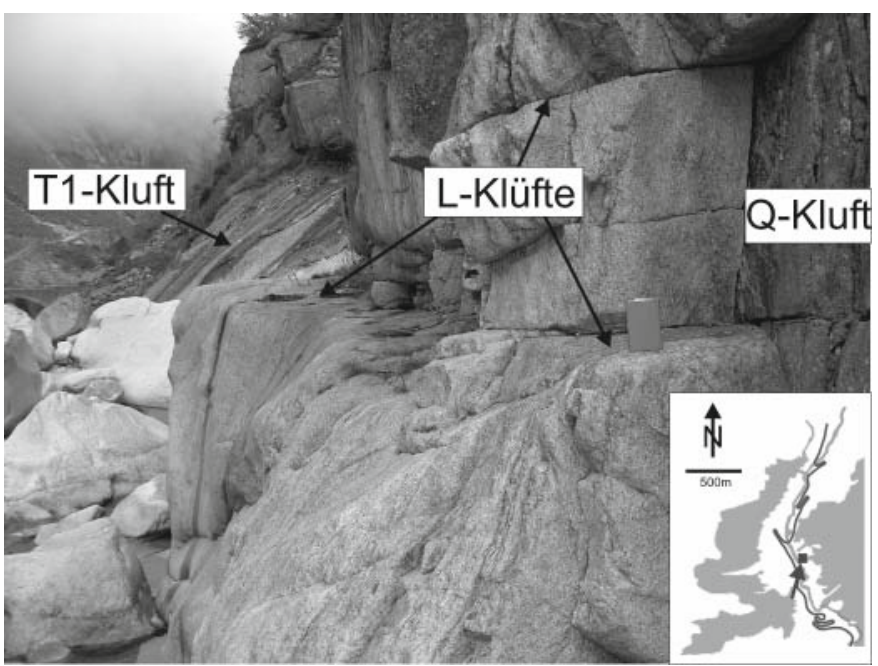

Fig. 8. Unterteilung der subhorizontalen Kluftfamilien anhand eines Aufschlusses auf der Ostseite des Fussbetts (vgl. kleine Karte). Neben den flach bis mittelsteil taleinwärts fallenden Klüften $\left(T_{1}\right)$ kann eine praktisch horizontal orientierte Kluftfamilie (L-Klüfte) sowie eine vertikale Q-Kluft beobachtet werden.

Im Gegensatz zu den über das gesamte Untersuchungsgebiet relativ einheitlich liegenden S- und Q-Klüften ist die Orientierung der „T“-Klüfte wie bereits erwähnt variabel. Die Talklüfte als dominierendes Set der Entlastungsklüfte fallen in den obersten noch begehbaren Teilen der Schöllenenschlucht vorwiegend zwischen 50 und $60 \mathrm{Grad}$ ein. Die im Bachbett gemessenen Klüfte sind meistens viel flacher (0 bis $40 \mathrm{Grad})$.
Neben dem Einfallen verändert sich auch das Fallazimut in Abhängigkeit der Topographie (Fig. 7c).

Bei der Kartierung der Störungen (Fig. 7d) konnten duktile Scherzonen (Mylonite) und spröd-duktile Störungen, d.h. Mylonite, welche anschliessend wieder in geringem Umfang durch spröde Deformation überprägt wurden (kataklastisch überprägte Mylonite), unterschieden werden. Häufig treten zudem stark geschieferte lamprophyrische Gänge auf, die teilweise kleine Scherbänder enthalten. Die Störungen streichen vorwiegend E-W bis NE-SW, untergeordnet NW-SE. Sämtliche Störungen sind steil einfallend. Die oft nur lokal aufgeschlossenen, teilweise nicht zugänglichen, aber aufgrund topographischer Anzeichen (markante Geländerunsen, enge Seitentäler) vermuteten Störungen, wurden anhand eines Luftbildes extrapoliert. Eine Detailbeschreibung der spröd überprägten duktilen Scherzonen des zentralen Aarmassivs findet sich in Laws (2001).

\section{Entlastungsklüfte an der Geländeoberfläche}

Die in Figur 5 mittels Stereoplots dargestellten „T-Klüfte“ erweisen sich bei genauerer Betrachtung im Feld als Überlagerung verschiedener Kluftfamilien mit ähnlicher lokaler Streichrichtung aber unterschiedlichem Einfallwinkel, d.h. aus mehreren sich im Aufschluss zum Teil schneidenden Kluftgenerationen. Figur 8 zeigt exemplarisch anhand eines Aufschlusses im Uferbereich der Reuss ein System von mittelsteil talwärts einfallenden Talklüften, welches gemeinsam mit einem Set von persistenten horizontalen Klüften und vertikalen Klüften (QKlüfte) auftritt. Die horizontalen Klüfte werden im Folgenden

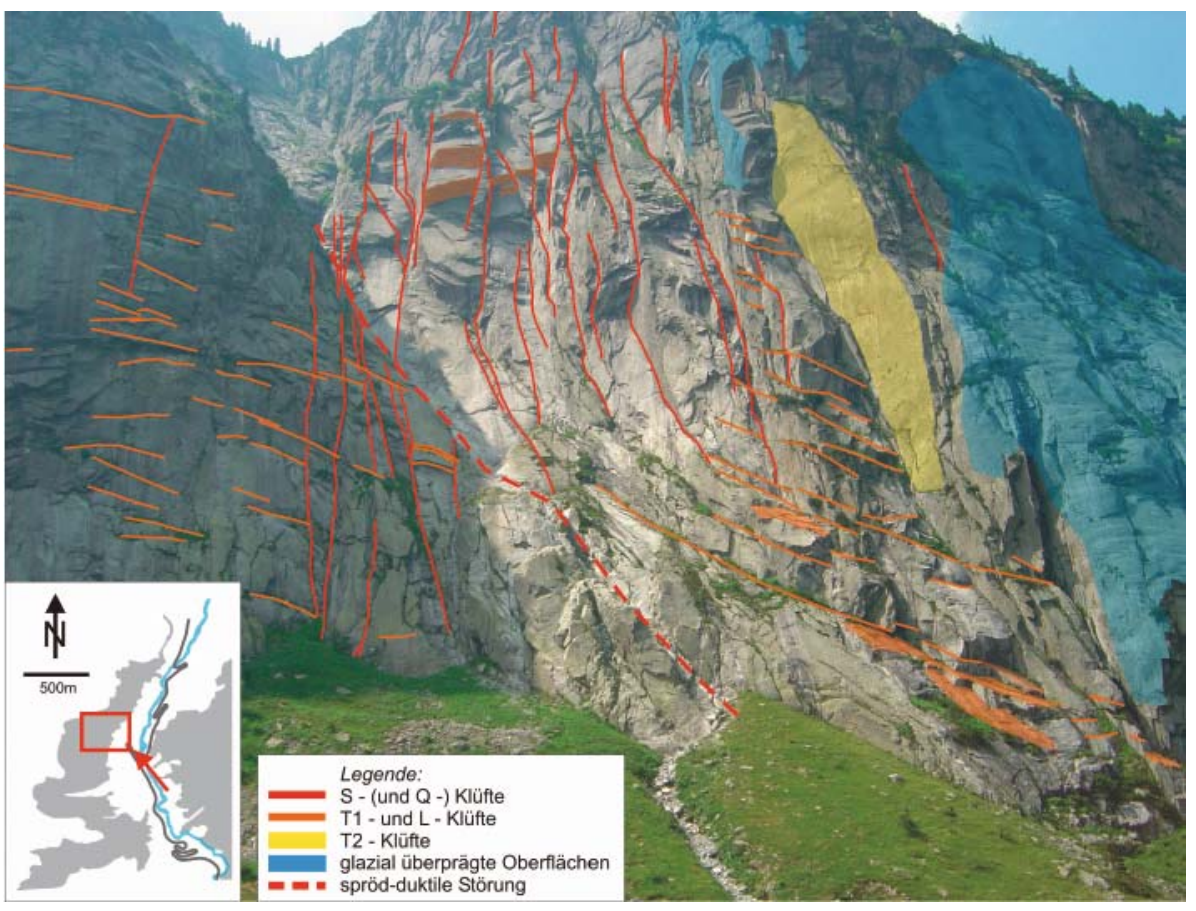

Fig. 9. Photographische Analyse der Trennflächen-Systeme in den unzugänglichen Felswänden der Schöllenenschlucht, Westseite, Teil Süd (Ausschnitt siehe Karte, Blickwinkel durch Pfeil markiert). Markierung der Spuren der S-, Q- und L-Klüfte sowie der Spuren oder Flächen der $\mathrm{T}_{1}$ und $\mathrm{T}_{2}$ Klüfte. Glaziale Erosionsoberflächen mit Schleifspuren sind blau markiert. 


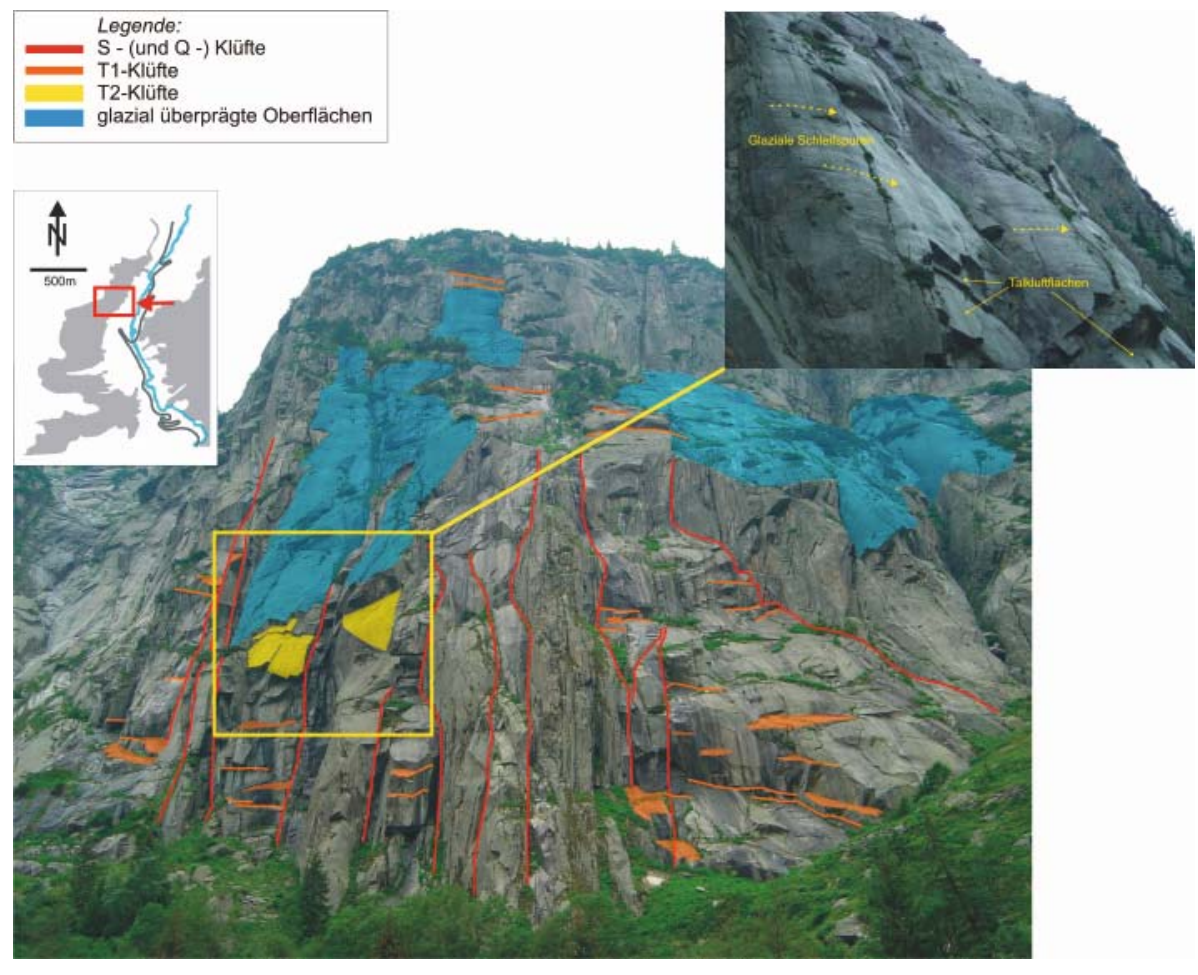

Fig. 10. Photographische Analyse der Trennflächen-Systeme in den unzugänglichen Felswänden der Schöllenenschlucht, Westseite, Teil Nord (gleiche Darstellung wie Figur 9). Auf dem Bildausschnitt können Talklüfte $\left(\mathrm{T}_{2}\right.$, gelb) beobachtet werden, welche sich unterhalb und parallel zu glazialen Erosionsflächen (blau) bildeten. in Anlehnung an die Nomenklatur von Cloos $(1921,1925)$ als L-Klüfte bezeichnet wird, jedoch ohne die entsprechende genetische Interpretation von Cloos zu implizieren.

Die Figuren 9-11 sind Fotos, die vom Gegenhang aus unterschiedlichen Blickwinkeln erstellt wurden, um die Raumstellung, Persistenzen und Interferenzen aller Kluftfamilien in den gut aufgeschlossenen, aber sehr steilen Felswänden der Schöllenen-Schlucht systematisch zu erfassen. Auf allen Figuren sind neben den Spuren und Flächen der Klüfte (rot, gelb, orange) sowie den glazialen Erosionsoberflächen (blau) auch die Bildausschnitte und Blickrichtung dargestellt (kleine Karte). Aufgrund der stärkeren Vegetation ist es auf der Ostseite der Schöllenen schwieriger, geeignete Aufschlüsse $\mathrm{zu}$ finden als auf der Westseite. Dennoch lassen sich auch hier die gleichen Kluftfamilien unterscheiden. Die Figuren 9-11 zeigen jeweils neben einem sehr steil einfallenden, der heutigen Topographie folgenden Talkluftsystem $\left(\mathrm{T}_{2}\right.$, gelb), ein zweites mittelsteil talwärts einfallendes Talkluftsystem $\left(\mathrm{T}_{1}\right.$, orange) auf.

In Figur 9 sind blau die glatten und abgerundeten Felsoberflächen hervorgehoben, auf denen systematisch horizontale Schleifspuren von Pleistozänen Gletschern vorhanden sind. Die hellgelb eingefärbte, steil talwärts einfallende Fläche ist eine $\mathrm{T}_{2}$-Talkluft mit einer vertikalen Ausdehnung von über hundert Metern. Auf dieser Kluftfläche können keine glazialen Schleifspuren festgestellt werden. Im zentralen Bereich der Figur 9 sind rot steil einfallende Klüfte eingezeichnet, welche hier primär Intersektionslinien der S-Klüfte darstellen. Zusätzlich verläuft durch Figur 9 eine spröd-duktile Störzone (kata- klastisch überprägter Mylonit, gestrichelt). In diesem Bereich sind keine grossen Talkluftflächen ausgebildet.

Figur 10 zeigt die Westseite der Schöllenen-Schlucht nördlich der Figur 9. Speziell hervorgehoben ist eine Stelle, wo direkt die geometrische Beziehung zwischen den glazial geprägten Erosionsflächen und $\mathrm{T}_{2}$-Talkluftflächen beobachtet werden kann (gelbes Viereck): Beide Flächen verlaufen parallel, die

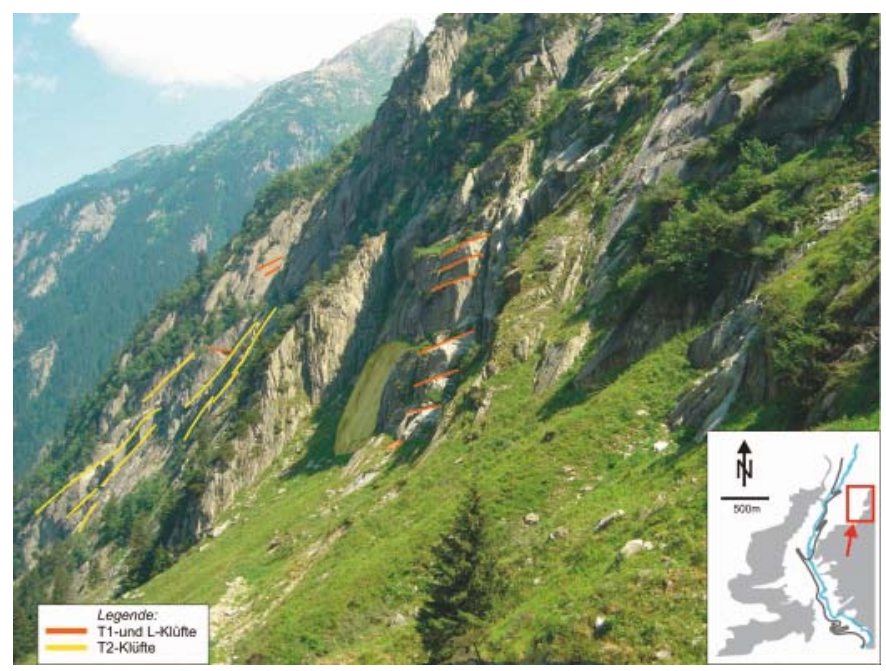

Fig. 11. Photographische Analyse der Trennflächen-Systeme in den unzugänglichen Felswänden der Schöllenenschlucht, Ostseite (gleiche Darstellung wie Figur 9).

410 S. P. Bucher \& S. Loew 


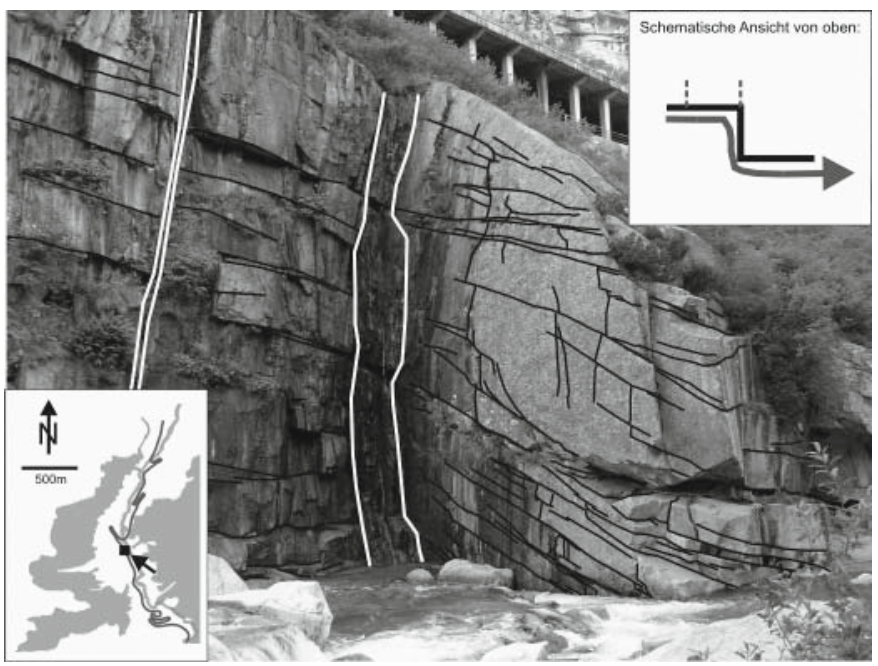

Fig. 12. Flach verlaufende, lokal gekrümmte Entlastungsklüfte (schwarz) im Bereich des Flussbetts mit zwei subvertikalen Störungen (leicht kataklastisch überprägte Mylonite; weiss).

Talklüfte werden durch junge und frische Anrisse freigelegt, die die glazialen Erosionsflächen diskordant abschneiden. Die rot eingefärbten S- (und Q-) Klüfte sind persistent, die orange eingefärbten $\mathrm{T}_{1}$-Klüfte schneiden diese nur selten.

Figur 11 wurde auf der östlichen Talseite aufgenommen. In der Bildmitte können orange eingezeichnet nun flach bis mittelsteil gegen West einfallende $\mathrm{T}_{1}$-Klüfte beobachtet werden. Auf der linken Bildseite sind die steileren, parallel zur Geländeoberfläche verlaufenden $\mathrm{T}_{2}$-Talklüfte (gelb) sichtbar.

Im Flussbett bietet sich ein anderes Bild. Die Klüfte trennen das Gestein in Platten, die beidseitig meistens flach gegen das Flussbett einfallen. Es konnten einzelne gebogene Kluftflächen beobachtet werden, deren Fallwinkel sich innerhalb weniger Meter um rund 20 Grad verändert. Ein vollständiges Umbiegen solcher Entlastungsklüfte von der einen Talseite auf die andere konnte aber nirgends direkt nachgewiesen werden, da der Fels meistens nur auf einer Flussseite aufgeschlossen und auf der anderen Seite mit Geröll und Vegetation bedeckt ist.

Einen schönen Aufschluss am westlichen Flussufer im oberen Bereich der Schöllenen zeigt Figur 12. Aufgrund der idealen räumlichen Anordnung erhält man einen dreidimensionalen Einblick in die Raumstellung der Trennflächen, Persistenzen und Kluftfrequenzen. Der Aufschluss ist rund $10 \mathrm{~m}$ hoch. Der Granit ist entlang der Entlastungsklüfte deutlich in flach einfallende, Dezimeter bis ungefähr Meter dicke Platten gespalten. Praktisch senkrecht dazu verlaufen Klüfte geringer Ausdehnung, welche meistens durch die flachen Klüfte begrenzt sind. Weiss eingezeichnet sind zwei subvertikale sprödduktile Störungen (leicht kataklastisch überprägte Mylonite). Auf der Skala des Untersuchungsgebietes zeigen sich grosse Unterschiede in den Kluftmustern von Aufschlüssen ähnlicher geomorphologischer Situationen (Fig. 8 und 12).

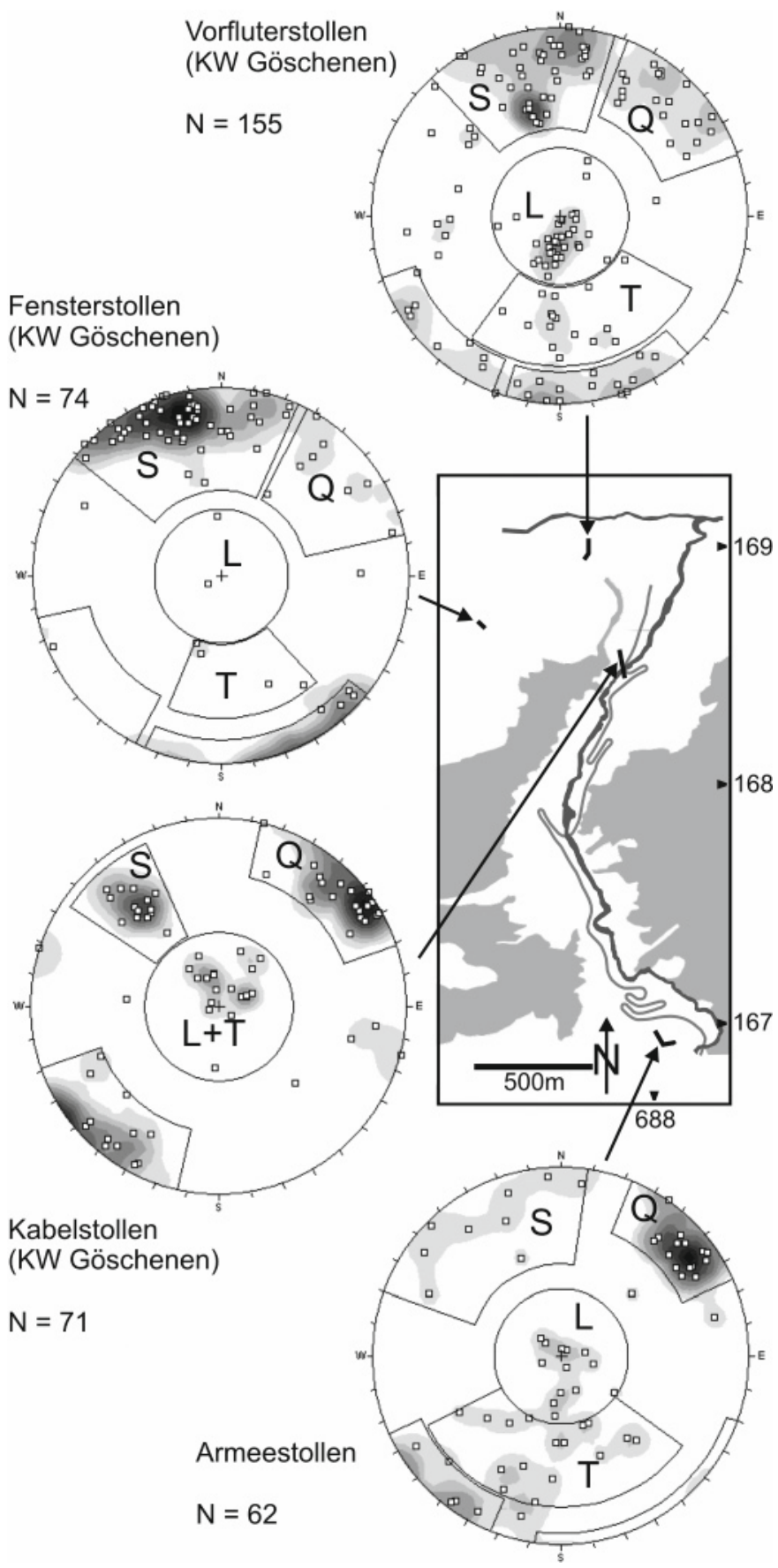

Fig. 13. Lage der Scanline-Messungen in Untertagebauwerken und Raumstellung der untertägig erfassten Klüfte. Die verschiedenen Kluftfamilien wurden in den Stereoplots manuell definiert.

\section{Scanline-Messungen in Stollenbauten}

Um einen besseren Einblick in die Tiefenentwicklung der verschiedenen Kluftsysteme und ihrer statistischen Parameter zu erhalten, wurden in drei Stollen des Kraftwerks Göschenen sowie in einem nicht mehr benutzten Armeestollen ScanlineMessungen der Trennflächen durchgeführt (nach der Methode 


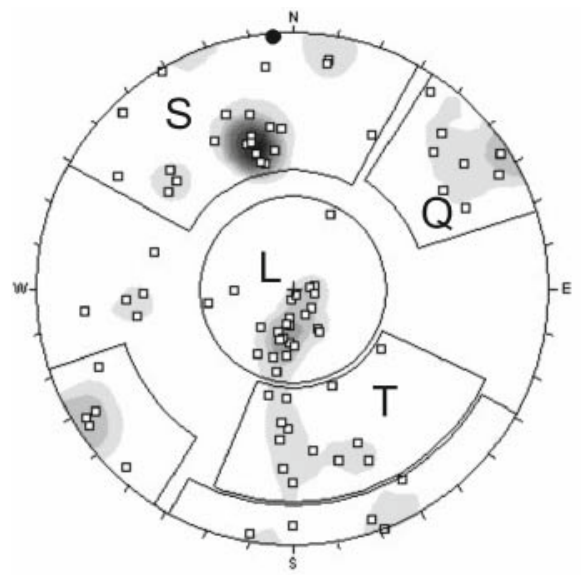

Vorfluterstollen Scanline 1 $\mathrm{N}=97$

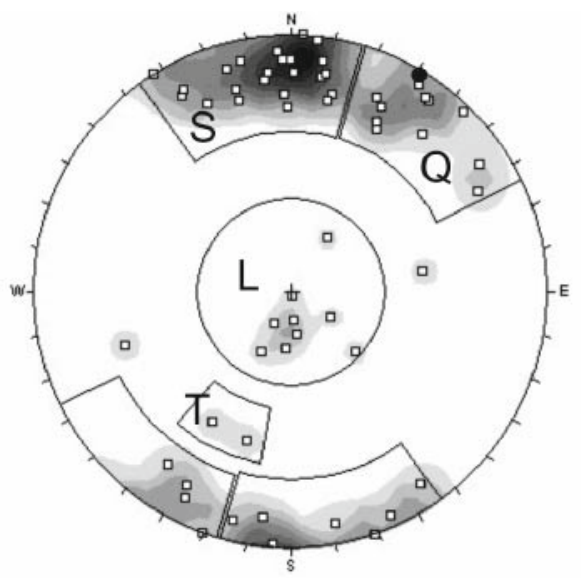

Vorfluterstollen Scanline 2 $\mathrm{N}=58$

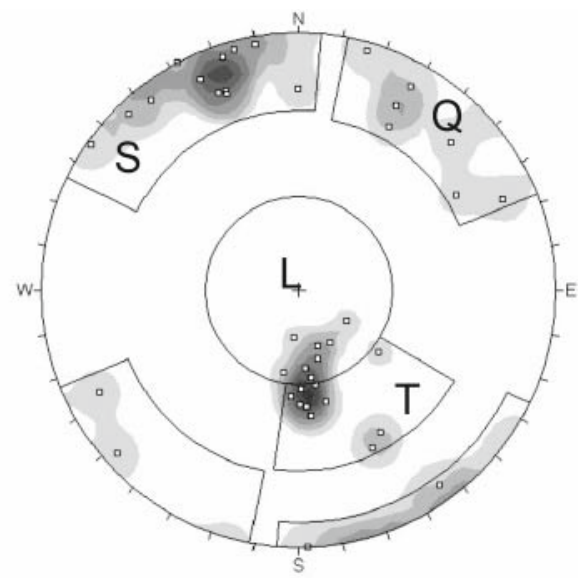

Messungen an Geländeoberfläche $\mathrm{N}=41$

Fig. 14. Stereoplots der Kluftmessungen im Vorfluterstollen des KW Göschenen und in der Umgebung an der Geländeoberfläche. Im linken und im mittleren Plot ist die Lage (Streichen) der Scanlines eingezeichnet (schwarze Punkte).

von Priest 1993). Einen Überblick über die Lage dieser Bauwerke und die in diesen erfassten Trennflächen gibt Figur 13.

Die umfangreichsten und aussagekräftigsten Messungen wurden im Vorfluterstollen des Kraftwerks Göschenen (KWG) ausgeführt. Der zuerst N-S-verlaufende Vorfluterstollen des KWG führt vom Portal im Göschenertal rund $160 \mathrm{~m}$ gegen das Berginnere und erreicht dort eine Überlagerung von ca. $90 \mathrm{~m}$. Zuhinterst dreht der Vorflutstollen nach SW ab, weshalb zwei verschiedene Scanlines durchgeführt wurden. Die Länge der ersten Scanline betrug $57 \mathrm{~m}$, die der zweiten tiefer unter Terrain gelegenen Scanline $18 \mathrm{~m}$. Der Stollen liegt knapp oberhalb des Flussniveaus der Göschener Reuss. Die vordersten $70 \mathrm{~m}$ sowie ein Abschnitt von rund $15 \mathrm{~m}$ zwischen den beiden Scanlines sind betoniert und lassen keine Messungen zu.

Figur 14 zeigt die Messungen der zwei durchgeführten Scanlines sowie die Messungen, die im Gebiet des Vorfluterstollens an der Geländeoberfläche gemacht wurden. Messungen an der Oberfläche waren aufgrund der starken Bewaldung nur beschränkt möglich. Berücksichtigt man die unterschiedlichen Orientierungen und Ausprägungen der alpinen Schieferung der Stollenabschnitte, so treten S-, Q-, und L-Klüfte in beiden Scanlines mit ungefähr gleicher relativer Häufigkeit auf. Die horizontalen L-Klüfte sind auch am Stollenende auffallend deutlich ausgeprägt. Die Talklüfte bilden sich im Vorflutstollen nach der lokalen Topographie im W-E-streichenden Göschenertal aus. Die Beobachtungen an der Geländeoberfläche dokumentieren - wie in der Schöllenen - zwei Generationen von Talklüften, welche hier nun mit 40-70 Grad gegen $\mathrm{N}$ einfallen. Jedoch ist eine Differenzierung dieser beiden Generationen von Talklüften im Stollen äusserst schwierig. Die Anzahl $\mathrm{T}_{1}$-und $\mathrm{T}_{2}$-Klüfte nimmt auf der ersten Scanline mit zunehmender Überlagerung ab (Fig. 15). Auf der zweiten Scanline konnte zusätzlich eine einzelne Talkluft rund $150 \mathrm{~m}$ im Berginnern bei einer Überlagerung von etwa 80 Metern nachgewiesen werden. Ähnliche
Beobachtungen beschreibt Stalder (1964) im Grimselgebiet, wo Talklüfte bei abnehmender Frequenz bis rund $200 \mathrm{~m}$ ins Berginnere nachgewiesen wurden.

Der Kabelstollen des KWG führt mit einer vertikalen Distanz von $20 \mathrm{~m}$ direkt unter der Reuss hindurch (Fig. 13). Neben den steil einfallenden S- und Q-Klüften können in diesem Bereich der Schöllenen-Schlucht analog zu den oben beschriebenen Aufschlüssen am Bachbett (Fig. 8 u. 12) insbesondere zahlreiche flach verlaufende Klüfte im vertikalen Abständen im dm-Bereich beobachtet werden.

Der Fensterstollen des KWG befindet sich auf rund $1400 \mathrm{~m}$ ü.M., also etwa $300 \mathrm{~m}$ über dem Talboden des Göschenertals. Die Scanline beginnt rund 10 Meter im Berginnern und ist $38 \mathrm{~m}$

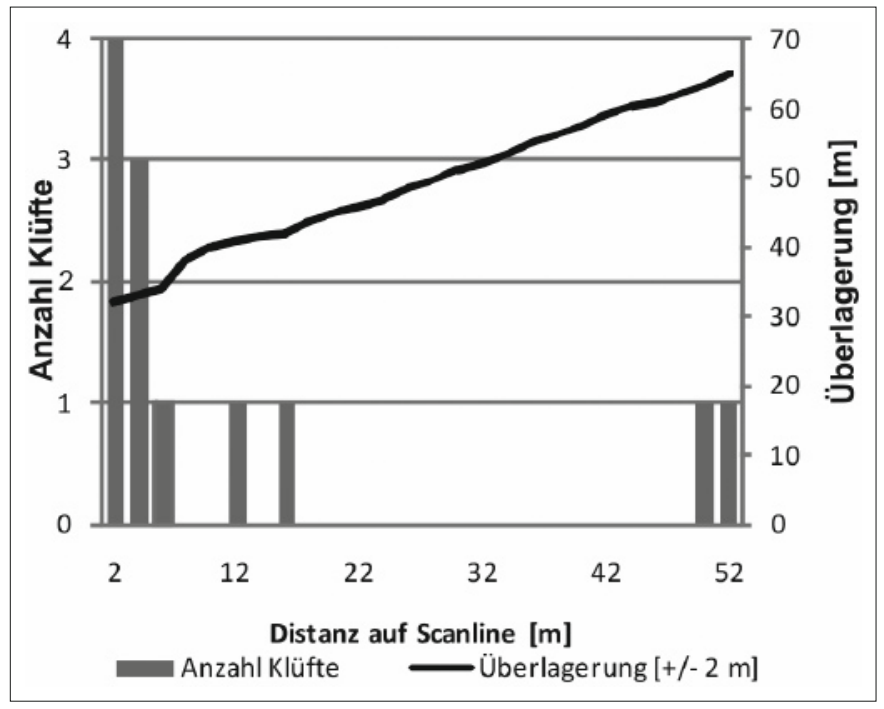

Fig. 15. Abnahme der Talklüfte mit zunehmender Distanz und Überlagerung im Vorfluterstollen des KW Göschenen. 


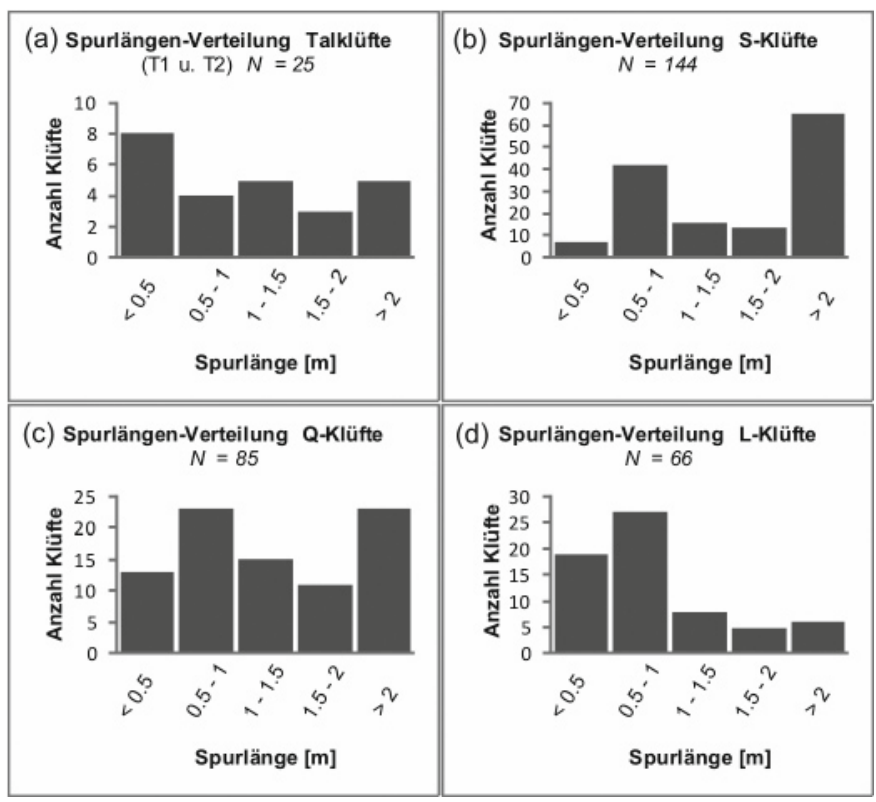

Fig. 16. Sichtbare Spurlängenverteilung der verschiedenen Kluftfamilien unter Einbezug sämtlicher durchgeführter Scanlines aus allen vier Stollen. Talklüfte (a), S-Klüfte (b), Q-Klüfte (c), L-Klüfte (d).

lang. Die Gesteinsüberlagerung nimmt von 12 auf $47 \mathrm{~m} \mathrm{zu}$. Der Stereoplot zeigt ein stark ausgeprägtes S-Kluftset (Fig. 13). Q-Klüfte schneiden die Scanline selten, da ihr Streichen subparallel zum Stollen verläuft. Die dadurch teilweise schön aufgeschlossenen Q-Kluftflächen sind oft mit einem feinen Chloritbelag überzogen (vgl. Fig. 6). Tal- und L-Klüfte können im Fensterstollen nur vereinzelt und unsystematisch beobachtet werden.

Der Armeestollen führt im oberen Bereich der Schöllenen ins Berginnere (Fig. 13). Da die Gesteinsaufschlüsse immer wieder durch betonierte Zwischenstücke unterbrochen werden, sind nur kürzere, nicht zusammenhängende Scanlines (insgesamt sechs, max. $22 \mathrm{~m}$ lang) möglich. Häufig können flach verlaufende L-Klüfte und parallel zur Geländeoberfläche orientierte Talklüfte beobachtet werden. Talklüfte konnten bis zum Ende der Messungen, $80 \mathrm{~m}$ ab Portal, nachgewiesen werden.

Die Spurlängenverteilung der verschiedenen Kluftfamilien ist in Figur 16 dargestellt. Dabei wurden die Messungen aus allen vier oben beschriebenen Stollen kombiniert. Da die Stollendurchmesser 2.5-3 m betragen, stellen Spurlängen grösser als $2 \mathrm{~m}$ nicht die wahren sondern nur messbaren Spurlängen dar. Dies ist insbesondere bei den S-Klüften von Bedeutung, welche vermutlich untertage die gleich grossen Spurlängen aufweisen wie an der Geländeoberfläche (Fig. 9 und 10). Die vorwiegend kleinen Spurlängen der T- und L-Klüfte weisen darauf hin, dass diese an der Geländeoberfläche ausgedehnter sind als in der Tiefenlage der hier untersuchten Untertagebauwerke (20-90 m vertikale Überdeckung).

\section{Fraktographische Untersuchung}

\section{Kluftstrukturen in Graniten}

Die Genese und die relativen Altersbeziehungen der im vorangegangenen Kapitel beschriebenen Kluftfamilien können durch fraktographische Untersuchungen besser verstanden werden. Die Tektono-Fraktographie (Bahat 1991) befasst sich mit der Beschreibung und Interpretation von spezifischen Oberflächenstrukturen auf Klüften, die während dem Bruchprozess entstehen (Fig. 17). Detailuntersuchungen zur Genese und Fraktographie von Klüften in Graniten wurden insbesondere von Cloos (1921), Bankwitz und Bankwitz (1995) und Bahat et al. (2005) publiziert. Auf einer Hauptkluftfläche können während dem Bruchprozess sogenannte Federmarken, Rissmarken und Kluftrandzonen gebildet werden (Twiss 1992). Diese sind wie folgt charakterisiert:

Federmarken (plumose markings): Regelmässiges Muster von feinen Federlinien (feather-like structures composed of barbs or hackles), welche sich von einem Punkt oder einer zentralen Achse gegen den Rand der Hauptkluftfläche ausbreiten (Fig. 18a). Der Punkt, an dem die Federlinien zusammenlaufen, entspricht dem Kluftursprung. Die Federlinien wiederspiegeln die Ausbreitungsrichtung des Bruchs. Die Bruchfront verläuft senkrecht zu den Federmarken. Eine langsame Bruchbildung (Grössenordnung 10-5 $\mathrm{m} / \mathrm{s}$; Bahat et al. 1999) begünstigt die Entwicklung von Federmarken. Sie kommen nur auf Dehnungsbrüchen vor, was sie von Scherbrüchen unterscheidet. Federmarken kommen in der Schöllenen relativ häufig vor.

Rissmarken (rip marks) oder Ringstrukturen: Kurvige, rampenförmige Strukturen, die ungefähr senkrecht zu den Federlinien verlaufen und zwei angrenzende subparallele Flächen der Kluftoberfläche verbinden (Fig. 18b). Rissmarken liegen konkav zum Ursprung des Bruchs und werden als Stellen interpretiert, wo die Bruchbildung zwischenzeitlich gestoppt wurde, weshalb sie manchmal auch als Stopmarken (arrest marks) bezeichnet werden. In der Schöllenen sind Rissmarken relativ selten zu beobachten.

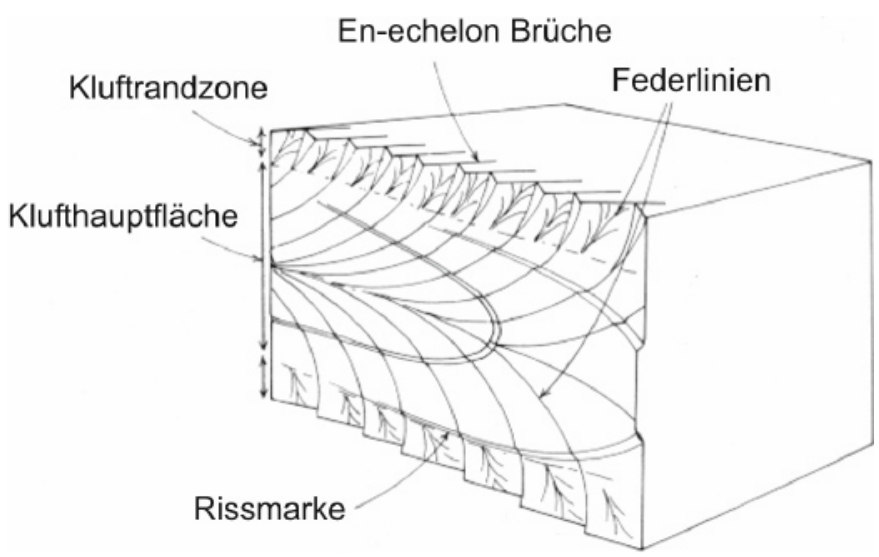

Fig. 17. Blockdiagramm mit verschiedenen fraktographischen Merkmalen auf Kluftoberflächen in einem geschichteten Sedimentgestein (aus Ramsay \& Huber 1987, nach Hodgson 1961). 

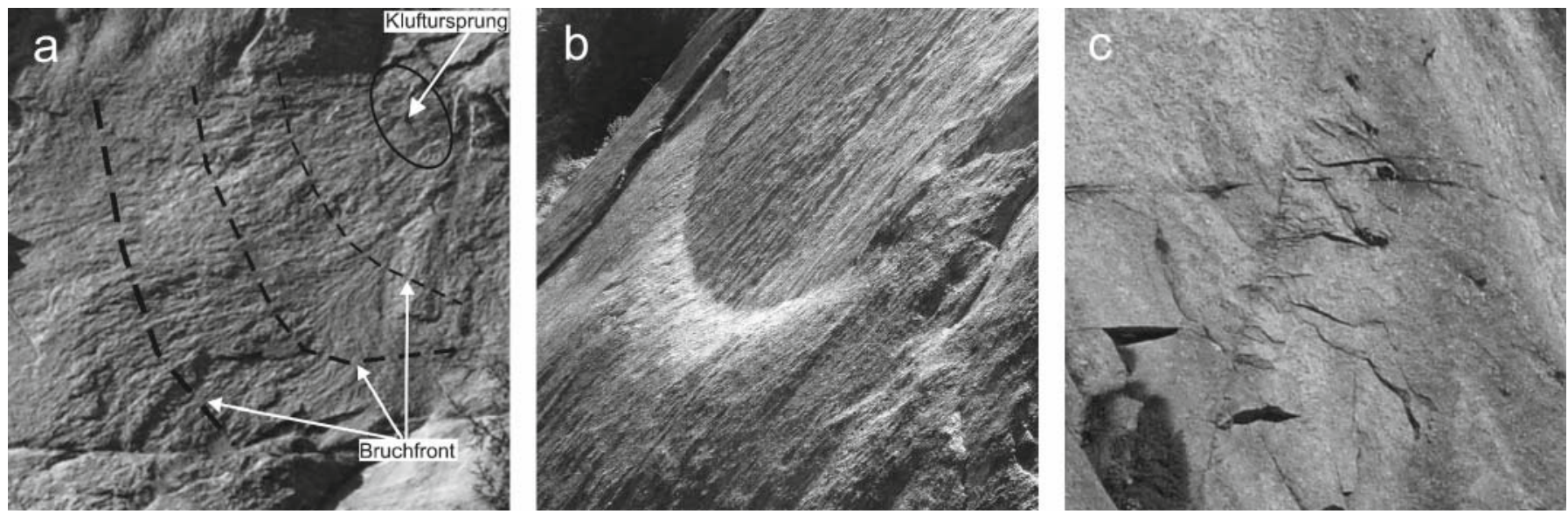

Fig. 18. Fraktographische Merkmale der Klüfte im Zentralen Aaregranit. (a) Geschwungene Federstruktur. Das Bild zeigt einen Ausschnitt von ca. $7 \times 7$ m. Der Kluftursprung ist oben rechts. Die Bruchfront verläuft senkrecht zu den Federlinien und verschiebt sich nach links unten. (b) Ca. $4 \mathrm{~m}$ breite Rissmarke. Deutlich sichtbar sind die senkrecht zur Rissmarke verlaufenden Federlinien. (c) Eine der wenigen schön ausgebildeten Kluftrandzonen. Die Kluftfläche endet an der steilen Kluft. Der Bildausschnitt beträgt ca. $1.5 \times 1.5 \mathrm{~m}$.

Kluftrandzonen (fringe composed of en-échelon fractures): Am Rand einer Hauptkluftfläche kann sich diese in eine Serie von Flächen aufteilen, welche leicht zur Hauptkluftfläche geneigt sind (twist hackles; Fig. 18c). Auf diesen geneigten Flächen können wiederum Federstrukturen ausgebildet sein (Fig. 17). Charakteristisch für diese Randzone ist eine raue Morphologie. Solche Randzonen sind in der
Schöllenen meistens nur schwach oder gar nicht ausgebildet.

\section{Fraktographische Beobachtung an Klüften in der Schöllenen}

Fraktographische Beobachtungen an Klüften sind stark von den Lichtverhältnissen und der Blickrichtung abhängig. Die

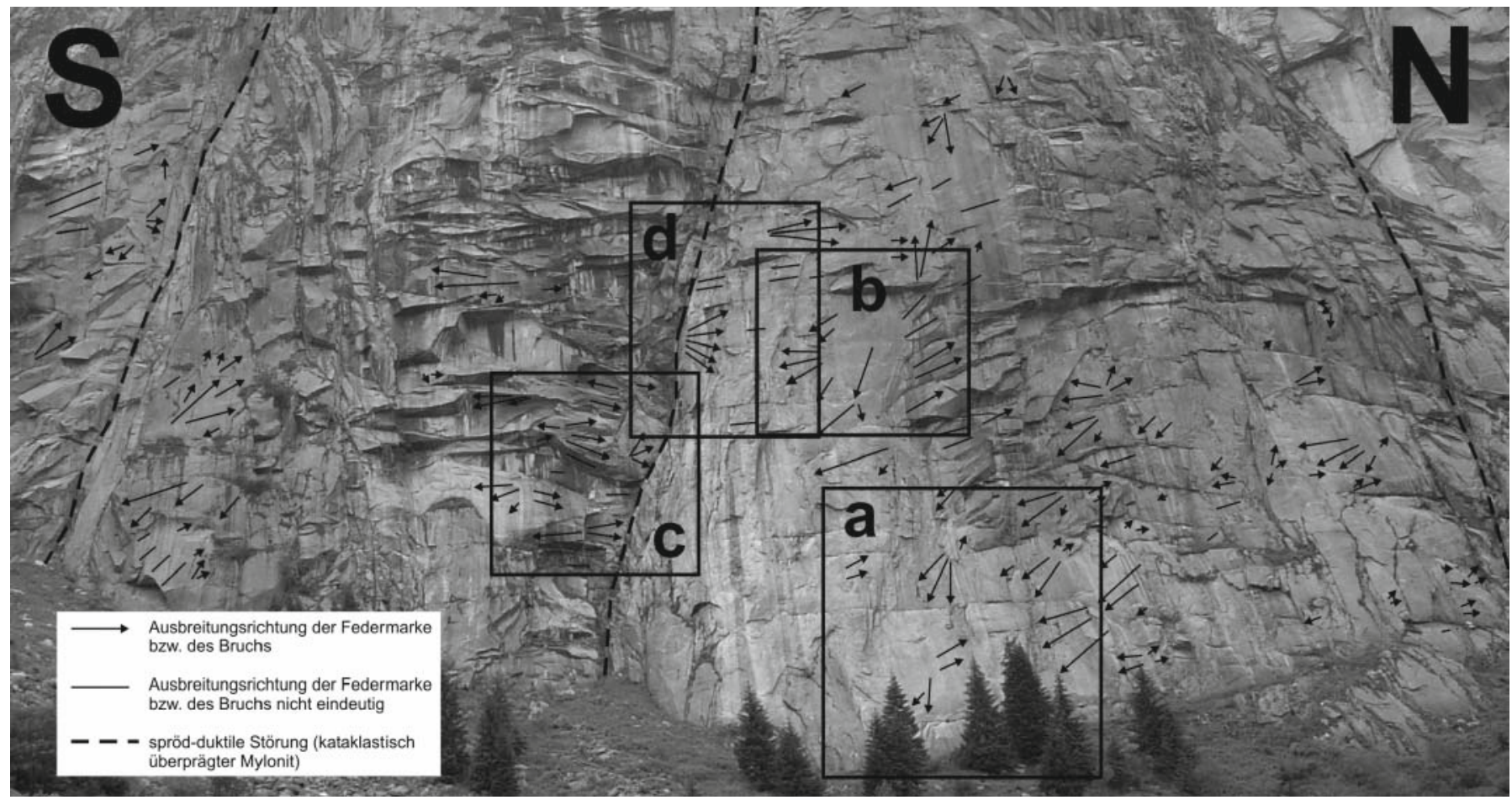

Fig. 19. Überblick der photographisch kartierten Oberflächenkluftstrukturen auf der westlichen Talseite zwischen den Koordinaten 687'397/167'972 und 687'361/167'731 mit Lage der in Figur 20 abgebildeten Detailausschnitte. Eingezeichnet sind die Ausbreitungsrichtungen aller Kluftbereiche mit Federmarken (Pfeile) sowie drei Störungen (kataklastisch überprägte Mylonite), welche offensichtlich einen Einfluss auf die Wandmorphologie und Kluftbildung haben. 

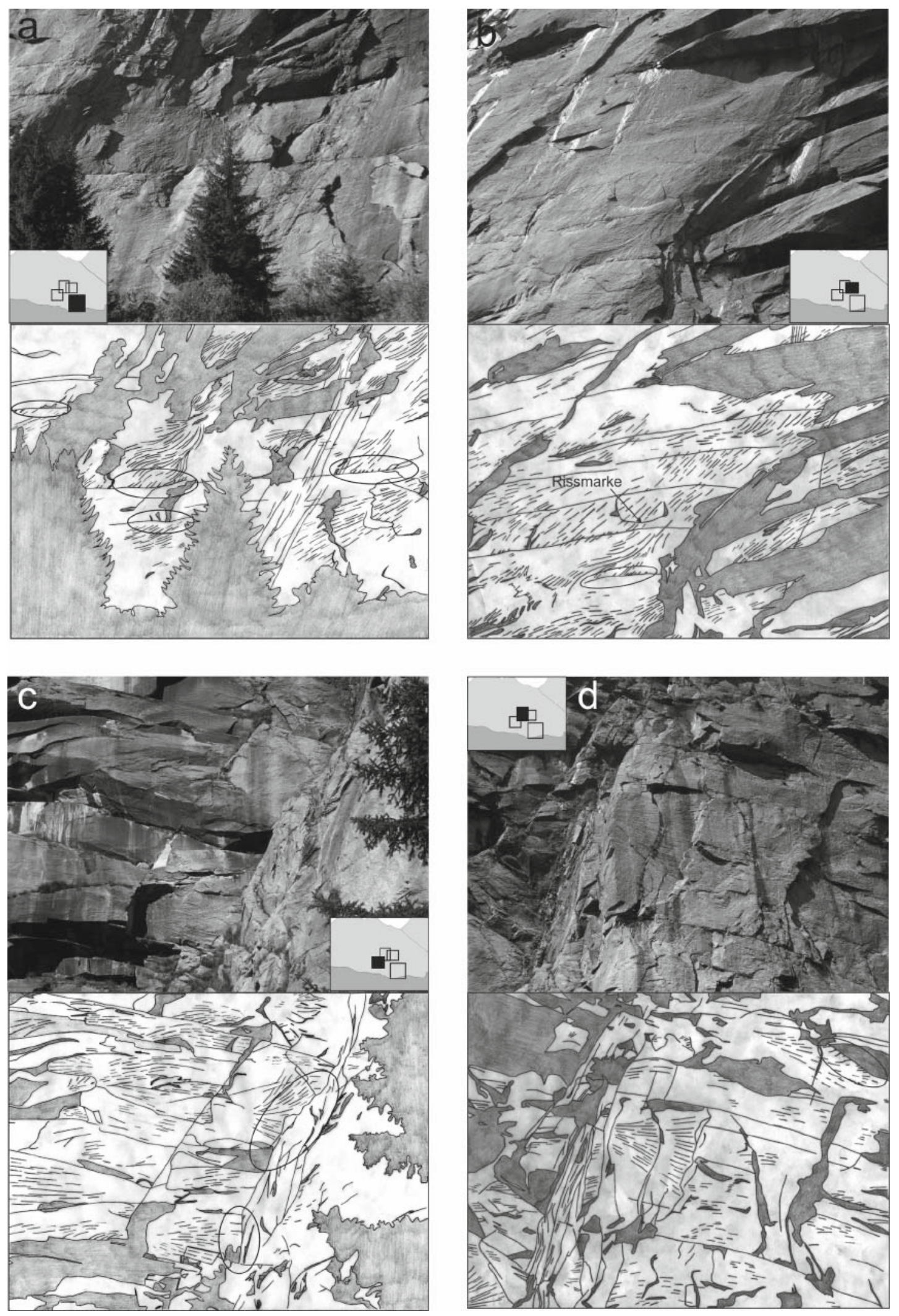

Fig. 20a-d. Vier Ausschnitte aus der Felswand der Talwestseite mit Federstrukturen auf Kluftoberflächen (jeweils Photo oben und dazugehörige Detailzeichnungen unten). Die Position der einzelnen Bilder ist in der Übersicht und in der Figur 19 dargestellt. Die Bildgrösse von Figur 20a beträgt ungefähr $40 \times 50 \mathrm{~m}$, jene der Figuren 20b-d je ca. $30 \times 40 \mathrm{~m}$. 
hier vorgestellten beeindruckend klaren fraktographischen Merkmale von Kluftoberflächen in der Schöllenen konnten im Spätherbst bei flacheinfallendem Schattenwurf herausgearbeitet werden. Detaillierte Analysen von Federmarken auf $\mathrm{T}_{2}$-Talklüften waren insbesondere auf der Westseite der Schöllenen zwischen den Koordinaten 687'397/167'972 und 687'361/167'731 möglich (Fig. 19). Der Aaregranit in diesem Abschnitt der Schöllenen besteht vorwiegend aus einem magmatischen Korngefüge mit schwacher metamorpher Mineraleinregelung (vgl. Fig. 4).

Die Pfeile in Figur 19 stellen die aus Federlinien von Federmarken abgeleiteten Ausbreitungsrichtungen der Entlastungsklüfte dar. Neben dem Bildungsmechanismus können aus den fraktographischen Merkmalen auch relative Altersbeziehungen der verschiedenen Kluftsysteme abgeleitet werden, da sich neue Brüche nicht über freie präexistente Kluftoberflächen ausbreiten können (Twiss 1992). Figur 20a-d zeigt exemplarisch vier Ausschnitte aus dieser Felswand, darunter jeweils die dazugehörige Detailzeichnung, auf welchen die häufigen Federmarken, seltenen Rissmarken und Kluftinterferenzen hervorgehoben wurden.

Figur 20a zeigt Details der Federstrukturen auf $\mathrm{T}_{2}$-Kluftoberflächen und ihre Interaktion mit subhorizontalen Klüften. Es können elf verschiedene Federmarken mit unterschiedlichen Ursprungspunkten beobachtet werden. Auffallend ist, dass die Federstrukturen die subhorizontalen Klüfte nicht schneiden und dort manchmal sogar eine schwache Kluftrandzone ausbilden (vgl. Ellipsen). Dies sind starke Hinweise darauf, dass die $\mathrm{T}_{2}$-Klüfte jünger als die flacheren ( $\mathrm{T}_{1}$ - oder $\mathrm{L}$-) Klüfte sind.

Die Felswand der Figur 20b lässt sich in zwei morphologisch deutlich unterschiedliche Bereiche aufteilen. Der linke Bildteil zeigt einen Ausschnitt einer grossen, mittelsteil talwärts einfallenden $\mathrm{T}_{2}$-Kluftfläche. Rechts dagegen ist die Wand stufenartig ausgebildet. Die Orientierung der Federmarken spricht eher dagegen, dass es sich dabei um eine Kluftrandzone handelt. Die nach oben offene Rissmarke deutet darauf hin, dass sich dieser Bruch in mehreren Phasen von oben nach unten ausbreitete. Die Ellipse markiert wiederum eine Stelle, wo eine Federmarke an einer subhorizontalen Kluft endet, während sie sich links der Kluft in geschwungener Form weiter nach unten ausbreitet. Im Bereich der gut ausgebildeten $\mathrm{T}_{2}$-Talkluftflächen können zahlreiche horizontal verlaufende Kluftspuren, jedoch keine steil einfallenden S- oder Q-Klüfte beobachtet werden.

Figur 20c zeigt wiederum den Übergang zweier morphologisch unterschiedlicher Bereiche der Felswand. Zwischen den beiden Bereichen verläuft eine steil einfallende, spröd reaktivierte duktile Störung (kataklastisch überprägter Mylonit) mit stärker geklüftetem Nebengestein. Im linken Bereich zeigt die Wand ein sehr unruhiges Relief. Horizontal verlaufen hier in relativ gleichmässigen Abständen stufenförmige Absätze und Vorsprünge. Auf den Kluftflächen können vorwiegend horizontal verlaufende Federmarken beobachtet werden, d.h. die Brüche breiteten sich in diesem Bereich der Felswand hauptsächlich horizontal aus. Die Ellipsen markieren Bereiche, wo die sich nach rechts ausbreitenden Federmarken gegen die steileinfallende Störung bzw. gegen das stärker geklüftete Nebengestein auffächern und gestoppt werden. Die $\mathrm{T}_{2}$-Klüfte sind darum klar jünger als die spröd-duktile Störung und die steilstehenden Klüfte.

Figur 20d zeigt den gleichen morphologischen Übergang an anderer Stelle (vgl. Situation Fig. 19), wobei dieses Bild aufgrund des Aufnahmewinkels stark verzerrt ist. Auf der rechten Seite der spröd reaktivierten Scherzone breiten sich die Federmarken wiederum nach rechts aus und fächern dabei auf. Auch die anderen Talklüfte breiten sich hier aufgrund der Federlinien primär in horizontaler Richtung aus. Die Ellipse zeigt eine der selten zu beobachtenden, schwach ausgebildeten Kluftrandzonen.

Auf der untersuchten Felswand konnten insbesondere auf $\mathrm{T}_{2}$-Talklüften mehrere Dutzend verschiedene Federmarken identifiziert werden. Die teilweise sehr deutlich ausgebildeten Federmarken haben grösstenteils Ausdehnungen von geschätzten 5 bis $10 \mathrm{~m}$. Einzelne längliche Federmarken können jedoch auch deutlich über $10 \mathrm{~m}$ messen. Rissmarken sind deutlich weniger zahlreich vorhanden und haben eine Sekantenlänge von rund 2 bis $5 \mathrm{~m}$. Kluftrandzonen wurden auf den Talkluftflächen äusserst selten beobachtet.

In Figur 19 sind die Ausbreitungsrichtungen aller Kluftbereiche zusammengefasst, welche Federmarken aufwiesen. Die in der Regel subhorizontale Ausbreitungsrichtung wird jeweils schematisch mittels Pfeil dargestellt. Bei den Linien ohne Pfeilspitze war die Ausbreitungsrichtung nicht eindeutig festzustellen. Die Ergebnisse stammen teilweise auch von Federmarken, die ausserhalb der in Figur 19 dargestellten Ausschnitte liegen. Während die Talklüfte praktisch keine sichtbaren Kluftrandzonen ausbildeten, war eine solche auf einer in einem Stollen aufgeschlossenen, mit einem feinen Chloritbelag überzogenen Q-Kluftfläche sehr ausgeprägt ausgebildet (vgl. Fig. 6).

\section{Mechanische Interpretation und Modellierung der Talkluftgenese}

\section{Geometrisches Modell der Entlastungsklüfte}

Die Figuren 19 und 20 zeigen, dass die Entwicklung der $\mathrm{T}_{2}$ Klüfte ein komplexer Vorgang ist und die grossen Talkluftflächen aus vielen kleineren Federstrukturen aufgebaut sind, die sich, ausgehend von verschiedenen Ursprüngen, in unterschiedliche primär subhorizontale Richtungen ausbreiten. Grosse Talkluftflächen mit einer Ausdehnung von mehr als hundert Meter bestehen somit aus vielen kleineren Einzelklüften in geometrischer (aber nicht mechanischer) Kontinuität.

Aufgrund der in den beiden vorangegangenen Kapiteln beschriebenen Beobachtungen kann ein schematisches Querprofil durch die Schöllenen mit drei Generationen von Entlastungsklüften entwickelt werden (Fig. 21). Die jüngsten Talklüfte $\left(\mathrm{T}_{2}\right.$-Klüfte) verlaufen in diesem Kluftmodell parallel zur Topographie. Es gibt Hinweise darauf, dass diese Talklüfte im Bereich des Talbodens umbiegen (vgl. Fig. 7c). Direkt nachge-

416 S. P. Bucher \& S. Loew 

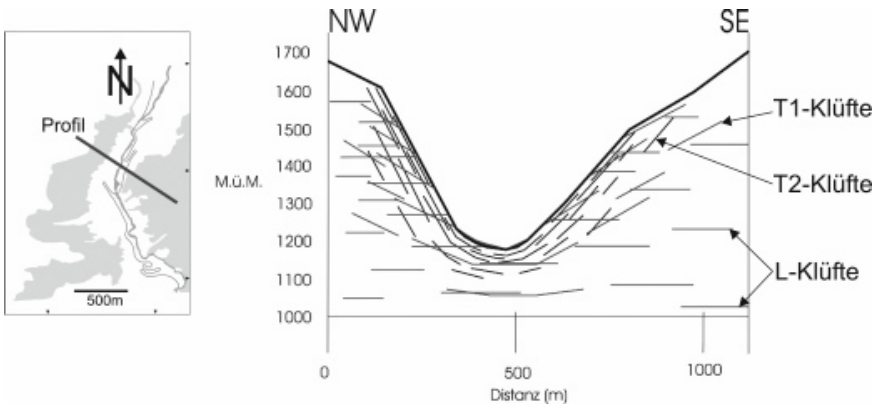

Fig. 21. Topographisches Querprofil durch die Schöllenen-Schlucht und vereinfachtes Modell der drei Sets von Entlastungsklüften. Links, Karte mit Lage der Profilspur.

wiesen werden konnte diese Umbiegung von der einen Talseite auf die andere aufgrund der Aufschlussverhältnisse aber nicht. Flacher, aber in ähnlicher Form und Streichrichtung verlaufen die $\mathrm{T}_{1}$-Klüfte. Praktisch horizontal und unabhängig von der Topographie verlaufen die L-Klüfte. Die Kluftabstände der $\mathrm{T}_{2^{-}}$ Klüfte nehmen tendenziell in den ersten $150 \mathrm{~m}$ unter Gelände zu, die L-Klüfte verändern ihre Abstände in den zugänglichen Stollenbauten und in natürlichen Aufschlüssen nicht systematisch. Der Tiefgang der $\mathrm{T}_{1}$-Klüfte ist unsicher.

Es ist zu vermuten, dass im Flussbett L-Klüfte in späteren Phasen reaktiviert wurden, respektive dass die drei oben aufgezeigten Entlastungskluftfamilien $\left(\mathrm{T}_{1^{-}}, \mathrm{T}_{2^{-}}\right.$und L-Klüfte) eine ähnliche Raumstellung haben; eine Zuordnung der einzelnen Klüfte zur jeweiligen Kluftfamilie ist hier kaum möglich.

Die grössten Unsicherheiten im Profil der Figur 21 birgt der Abschnitt zwischen dem Flussbett und dem Beginn der steilen Felswände in der Schöllenen. Dazwischen liegen meistens rund 100 bis 200 Meter Gehängeschutt und Vegetation. Weiter gilt es zu berücksichtigen, dass dieses schematische Profil vor allem im Bereich der steilen Felswände auf Fernbeobachtungen und nicht auf direkten Aufschluss-Messungen beruht.

\section{Bildungsmechanismus und Bruchkriterien der Entlastungsklüfte}

Figur 22 zeigt nach Holzhausen \& Johnson (1979) und Bahat et al. (1999) schematisch die Entstehung von longitudinalen Extensionsrissen in einem zylindrischen Testkörper ohne seitliche Einspannung. Bei gleichmässiger Probenbelastung in axialer Richtung können sich innerhalb der Probe aus kleinen Heterogenitäten (a) kleine fächerartige Kluftstrukturen (b) bilden. Durch Krümmung des Probenkörpers (c) ergeben sich Zugspannungen, welche zu einer progressiven Rissausbreitung führen. Durch die Interaktion der kleinen fächerartigen Strukturen ergibt sich schliesslich ein durchgehender longitudinaler Spaltbruch in Richtung der maximalen Belastung (oder genauer: normal zur minimalen Belastung). Ein Blick auf den um 90 Grad gedrehten Testkörper im Stadium (b) zeigt fächerartige Strukturen, die sich noch nicht $\mathrm{zu}$ einem durchgehenden Bruch zusammengeschlossen haben.
Detaillierte Labor-Analysen zur Entstehung von Extensionsrissen unter ein- oder zweiaxialer kompressiver Belastung wurden an homogenen Proben (z.B. Read et al. 1998, Eberhardt et al. 1999) und Proben mit „Griffith Cracks“ (z. B. Hoek \& Bieniawski 1965) ausgeführt. Die meisten dieser Studien wurden im Zusammenhang mit der Untersuchung von hohlraumparallelen Brüchen (Abschalungen und Bergschläge) in tieferen Untertagebauwerken (Endlagerstollen, Minen) ausgeführt. Nach diesen Arbeiten beginnen sich Abschalungen um tiefliegende Untertagebauwerke ab einem minimalen deviatorischen Spannungsniveau $\left(\sigma_{1}-\sigma_{3}\right)$ zu entwickeln, welches in Graniten etwa in der Höhe von $30-45 \%$ der einaxsialen Druckfestigkeit liegt (z.B. Read et al. 1998, Diederichs 2003). Dieses Spannungsniveau korreliert mit dem Beginn der Mikrorissbildung in Laborversuchen. Basierend auf Laborversuchen am Lac du Bonnet Granit setzen Eberhardt et al. (2004) für die Initialisierung von Entlastungsklüften im Mattertal (Randa) ein Mohr-Coulomb-Kriterium mit sehr tiefen Festigkeiten an (10 MPa Kohäsion, $5^{\circ}$ Reibung, $1 \mathrm{MPa}$ Zugfestigkeit).

Laboruntersuchungen von Hoek \& Bieniawski (1965) zeigen, dass die Grösse propagierender „Griffith Cracks“ in einem kompressiven Spannungsfeld primär vom Verhältnis der maximalen $\left(\sigma_{1}\right)$ zur minimalen $\left(\sigma_{3}\right)$ Hauptspannung abhängt: bei kleinen minimalen (kompressiven) Hauptspannungen und hohen maximalen Hauptspannungen entwickeln sich relativ grosse Extensionsrisse. Nach Hoek (1968) und Hoek \& Bieniawski (1965) entwickeln sich grosse Risse durch stabile Risspropagation insbesondere ab einem $\sigma_{3} / \sigma_{1}$-Verhältnis von
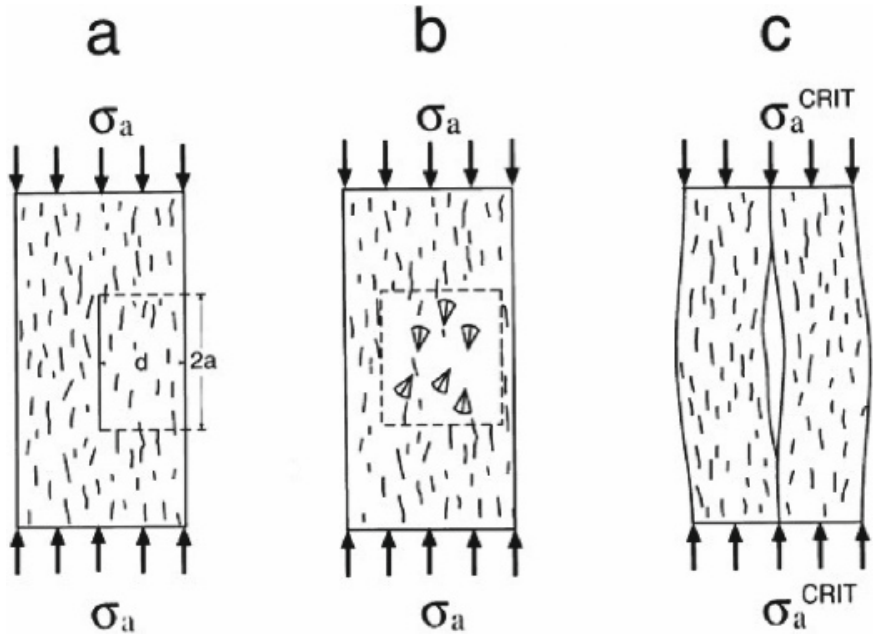

Fig. 22. Schematische Darstellung der Entwicklung eines axialen Spaltrisses in einer zylindrischen Probe unter einaxialer Belastung. Kleine initiale Heterogenitäten (flaws) innerhalb des Probenkörpers (a) führen zur Bildung früher fächerförmiger Risse (b). Diese ersten axialen Risse vereinigen sich unter der aufgebrachten Spannung $\sigma_{\mathrm{a}}$ zu einem grösseren Spaltriss mit der Länge 2a und dem Abstand d von der freien Oberfläche. Wird die Probe um $90^{\circ}$ gedreht, erkennt man die frühen Fächerstrukturen, welche sich nach oben und unten ausbreiten, sich jedoch noch nicht zusammengeschlossen haben (b). Die weitere Belastung und Verformung führt zur Krümmung der Probenteile (c) und zur Propagation des Spaltrisses durch die gesamte Probe (Holzhausen \& Johnson 1979, modifiziert durch Bahat et al. 1999). 

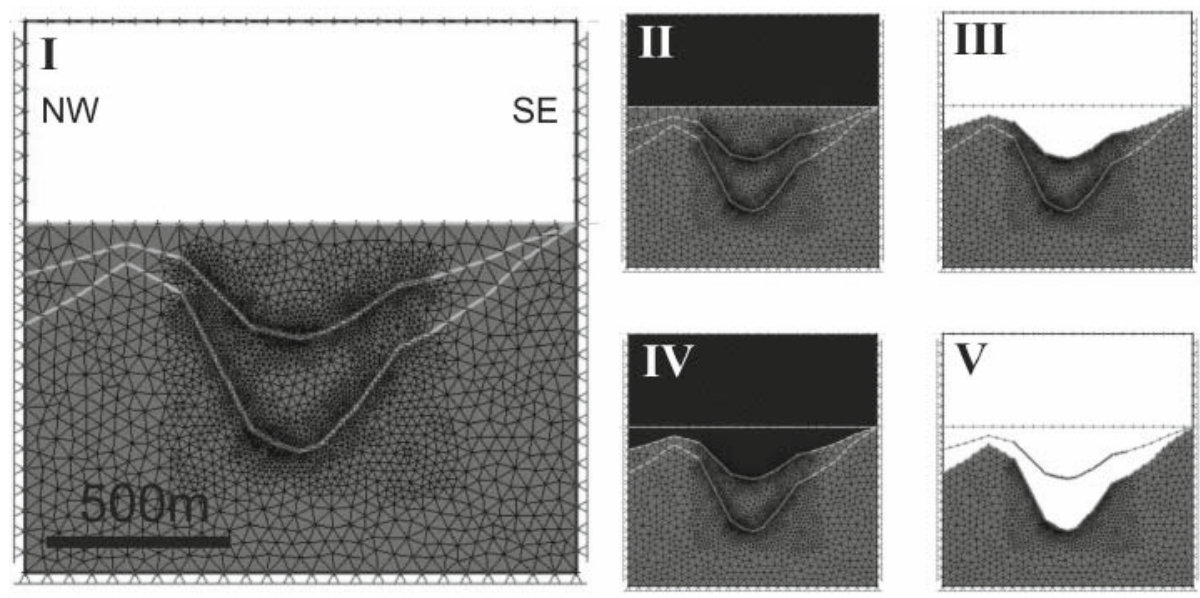

Fig. 23. 2D Finite Element Modell bestehend aus fünf hypothetischen Stadien der Pleistozänen Geländeentwicklung in der Schöllenenschlucht entlang dem Schnitt von Figur 21. Grau dargestellt ist das granitische Gebirge, dunkelgrau Eis und weiss Luft (für Erklärung siehe Text).
0.05 und kleiner. Sekundär hängt die Grösse dieser Extensionsrisse von der Grösse der initialen Heterogenität im Ursprung der Kluftbildung ab.

Wie im vorangegangenen Kapitel und in Laborversuchen gezeigt wurde, entwickeln sich die Talklüfte parallel zur Geländeoberfläche aus der Vereinigung mehrerer Fächerstrukturen. In Oberflächennähe und ausserhalb aktiver Trennflächen sind die Spannungsverhältnisse stark durch die lokale Topographie geprägt. Es ist somit davon auszugehen, dass die maximalen Hauptspannungen im Bildungsbereich der Talklüfte nahezu hangparallel liegen und sich analog zu den Laborverhältnissen Zugrisse parallel zur freien Oberfläche entwickeln können (Müller 1962). Die fraktographischen Merkmale (Federstrukturen) passen $\mathrm{zu}$ einem solchen Extensionsrissmodell (Bahat et al. 1999, 2005), stärkere Scherverformungen hätten diese Merkmale zerstört.

Mit zunehmender Tiefe unter Geländeoberfläche erhöhen sich die minimalen Hauptspannungen. Gemäss der Theorie von Hoek \& Bieniawski (1965) sollten sich mit zunehmender Tiefe die Grössen der Talklüfte verkleinern. Dies wird durch die Befunde der Scanline-Messungen in den untersuchten Stollen unterstützt, auch wenn eine belastbare Aussage $\mathrm{zu}$ den tiefenabhängigen Kluftgrössen heute nicht möglich ist.

\section{Modellrechnungen zur Entwicklung der Entlastungsklüfte}

Mit dem Finite Elemente Modell Phase ${ }^{2}$ (RocScience 2009) wurden entlang dem Schnitt von Figur 21 erste, stark vereinfachte 2-dimensionale numerische Berechnungen zum räumlichen Auftreten der $\mathrm{T}_{1}$ - und $\mathrm{T}_{2}$-Entlastungsklüfte durchgeführt. Das Ziel der Modellierung bestand darin, die möglichen Spannungsverhältnisse in der Schöllenen abzubilden und mit verschiedenen Bruchbedingungen von Extensionsrissen zu vergleichen. Dabei war auch von Interesse, ob neben den jüngsten $\mathrm{T}_{2}$-Talklüften zusätzlich eine frühere Generation von Talklüften, welche parallel zu einer früheren Topographie orientiert sind, modellmässig erklärt werden kann. Dazu wurden die Spannungsverhältnisse in der Schöllenen während und zwischen den Eiszeiten unter Berücksichtigung der Wasserdrücke berechnet und mit den oben diskutierten Bruchkriterien verglichen.

Da der Spannungszustand an Ende des Pleistozäns von der früheren Felsüberlagerung und der Spannungspfadentwicklung abhängig ist, wurde die Pleistozäne Erosionsgeschichte stark vereinfacht mit fünf aufeinanderfolgenden Modell-Stadien nachgebildet (Fig. 23), die die progressive Bildung des heutigen Reliefs grob vereinfacht darstellen. Die maximale Höhe der Eisoberfläche betrug gemäss Florineth \& Schlüchter (1998) während der letzten Eiszeit auf der Höhe des Querprofils in der Schöllenen etwa $2350 \mathrm{~m}$. Die maximale Eisüberlagerung im Modell beträgt demnach 550 bis 1170 m, je nach Entwicklungsstand des Reliefs in den verschiedenen Stadien. Die heutigen in-situ Spannungen sind stark von der initialen Gebirgsüberlagerung und dem Seitendruckbeiwert abhängig. Als Basisfall wurde von einer ebenen Topographie auf der Höhenlage der heutigen Bergspitzen (2900 m) und einem Verhältnis Horizontalspannung zu Vertikalspannung von 1 ausgegangen (Modellstadium 1). Das Stadium 2 simuliert eine Eiszeit mit einem Gletschervorstoss und einer ersten Taleintiefung. Das Stadium 3 zeigt ein Interglazial mit schwach entwickeltem Talrelief (entsprechend den Bildungsbedingungen der $\mathrm{T}_{1}$-Klüfte). Im Stadium 4 wird das Gebiet wiederum durch einen Gletscher mit einer maximalen Eisdicke von $860 \mathrm{~m}$ überfahren und eingetieft. Nach dem Gletscherrückzug wird mit Stadium 5 der heutige Zustand und die Bildungsbedingungen der $\mathrm{T}_{2}$-Klüfte erreicht.

Für die einaxiale Druckfestigkeit des Zentralen Aare Granits liegen 22 Proben des Bauprojekts des Gotthard-Basistunnels vor (Kobel \& Partner AG 1999), welche bei einem Mittelwert von $152 \mathrm{MPa} / \mathrm{m}^{2}$ primär im Bereich zwischen 100 und $200 \mathrm{MPa} / \mathrm{m}^{2}$ streuen. Dies ergibt ein minimales deviatorisches Spannungsniveau von etwa $35 \mathrm{MPa}$ für den Beginn der Mikrorissbildung. Als Young's Modul ergeben 11 Proben des gleichen Projekts einen Mittelwert von $39 \mathrm{GPa} / \mathrm{m}^{2}$. Die Poissonzahl wurde mit 0.3 modelliert.

418 S. P. Bucher \& S. Loew 

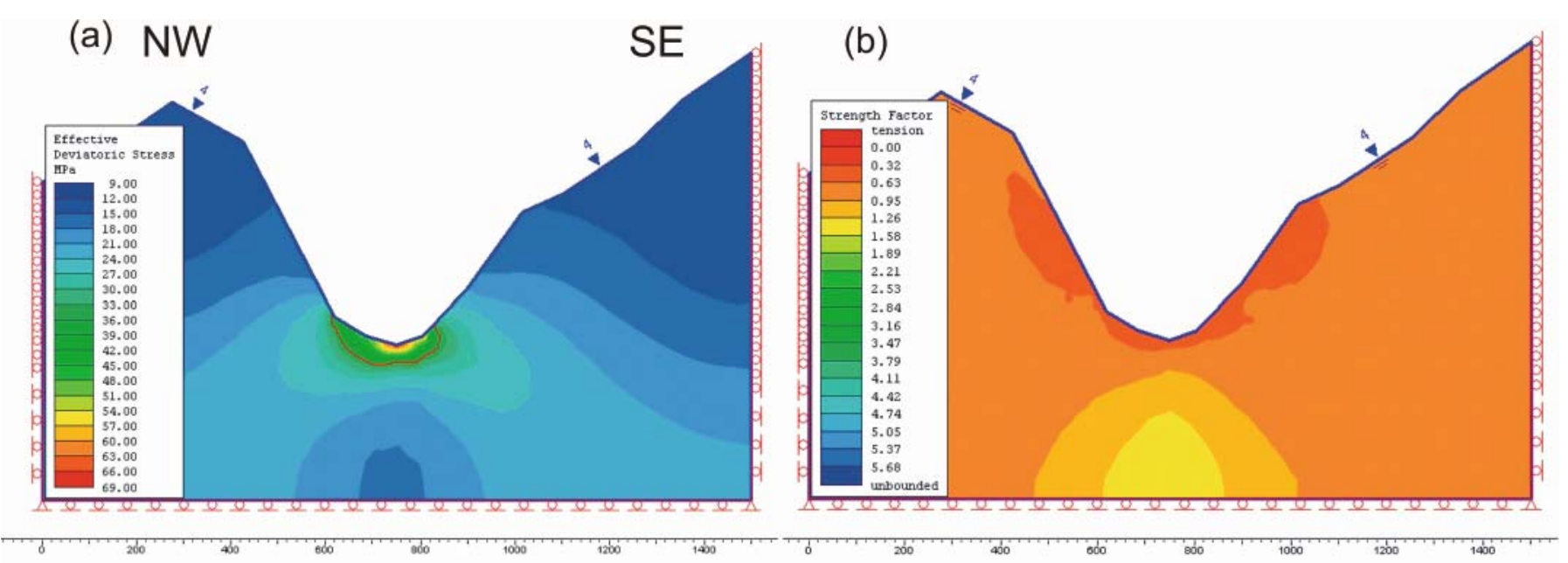

(c)

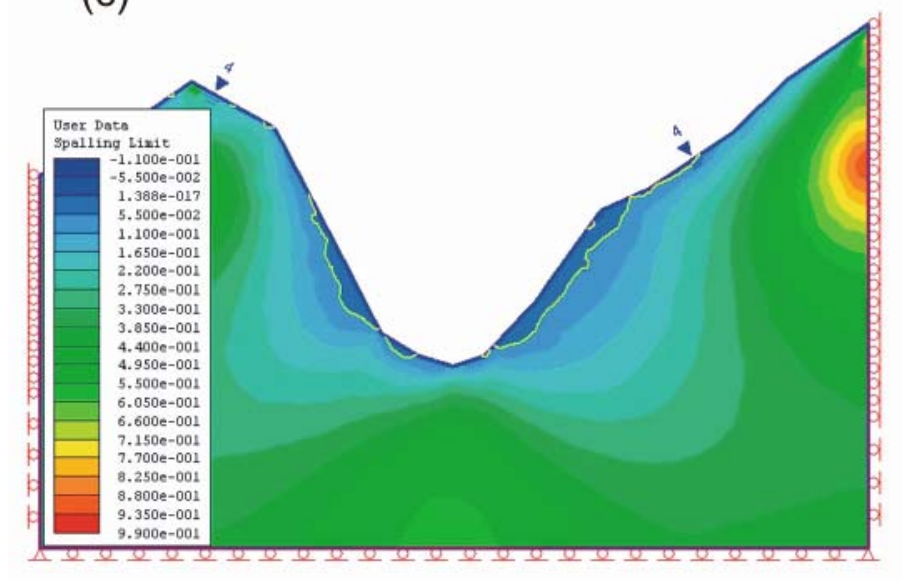

(d)

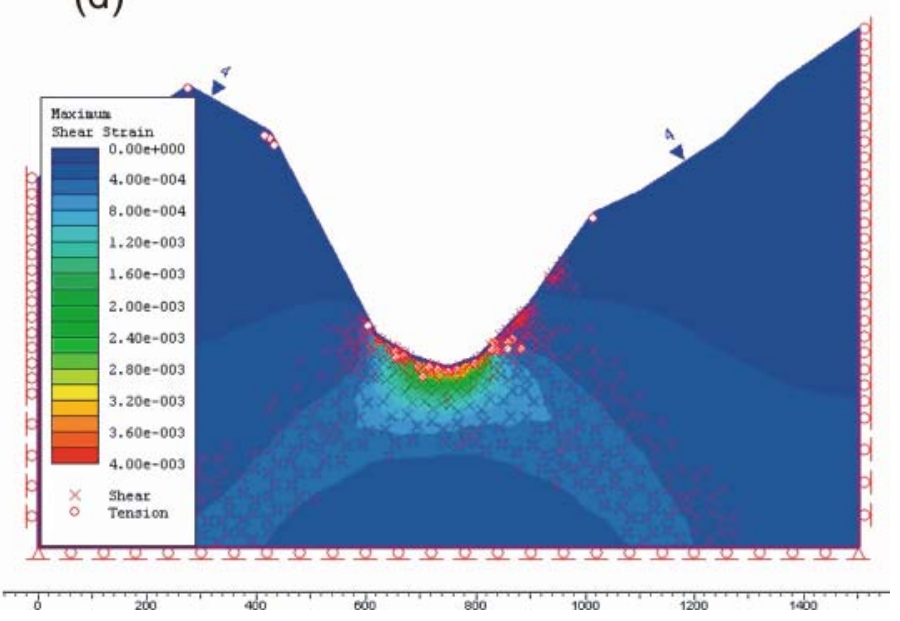

Fig. 24. Elastische (a, b, c) und elasto-plastische (d) Modellresultate für Stadium 5 (heutige Topographie). Dargestellt sind die simulierten Deviatorspannungen (a), Festigkeitsfaktoren (b), $\sigma_{3} / \sigma_{1}$-Spannungsverhältnisse (c) und die maximalen Scherverformungen inklusive Elementen mit plastischem Versagen (d). Als Bildungskriterien der Entlastungsklüfte wurden die Grenzlinien der Mikrorissbildung von $35 \mathrm{MPa}$ Deviatorspannung (a), die $\sigma_{3} / \sigma_{1}-$ Spannungsverhältnisse (spalling limit) von 0.05 (c) sowie die Mohr-Coulomb-Bruchkriterien gemäss Eberhardt et al. (2004) (Fig. 24b und d) angesetzt. Die blauen Dreiecke an der Geländeoberfläche entsprechen dem in Stadium 5 vereinfachend an der Geländeoberfläche angenommenen Grundwasserspiegel.

Figur 24 zeigt die Modellresultate für das Endstadium 5 sowohl für eine rein elastische Modellierung $(\mathrm{a}, \mathrm{b}, \mathrm{c})$, wie auch für eine elasto-plastische Modellierung (d). Die Tiefe der beobachteten Bildung von Talklüften kann mit dem Kriterium der maximalen $\sigma_{3} / \sigma_{1}$ Hauptspannungsverhältnisse $(0.05)$ überzeugend nachgebildet werden (Fig. 24c). Für die obigen Spannungsannahmen und das Kriterium, dass sich erste Rissbildungen ab einer Deviatorspannung von 35\% der einaxialen Druckfestigkeit entwickeln, bilden sich erst im letzten Stadium 5 und nur im Talboden der Schöllenen Entlastungsklüfte (Fig. 24a). Dies ist nicht konsistent mit den Feldbeobachtungen. Für die MohrCoulomb-Bruchkriterien gemäss Eberhardt et al. (2004), d.h. $10 \mathrm{MPa}$ Kohäsion, $5^{\circ}$ Reibung und $1 \mathrm{MPa}$ Zugfestigkeit, ergeben sich Zugrisse (Fig. 24d) in einem ähnlichen Bereich wie Figur 24a und kritische Spannungsverhältnisse (Fig. 24b) in einem ähnlichen Bereich wie Figur 24c. Der Festigkeitsfaktor einer elastischen Berechnung (Fig. 24b) stellt das Verhältnis der lokalen Festigkeiten zu den lokalen effektiven Spannungen dar. Bei einer Erhöhung der initialen Gebirgsüberlagerung oder der Seitendruckbeiwerte vergrössert sich der Bildungsbereich der Entlastungsklüfte, dafür wird aber die Tiefenverbreitung der Entlastungsklüfte gegenüber den Beobachtungen zu gross.

\section{Diskussion und Synthese}

Aufgrund der detaillierten strukturellen und fraktographischen Beobachtungen in der Schöllenen kann ein belastbares geometrisches und kinematisches Entwicklungsmodell der verschiedenen Generationen von Entlastungsklüften im zentralen Aaregranit abgeleitet werden. Im Gegensatz zur Darstellung in verschiedenen geomorphologischen Studien (Savage et al. 1985, Miller \& Dunne 1996) entwickeln sich 
Talklüfte vermutlich nicht als Scherbrüche, sondern als Extensionsklüfte in einem kompressiven Spannungsfeld. Die Kriterien von Extensionsbrüchen unter geringen Manteldrücken, wie sie für tiefe Tunnelbauwerke in granitoiden Gesteinen aus Laborversuchen abgeleitet wurden, führen zu neuen Ansätzen zur Modellierung von Talklüften. Dabei bestehen allerdings noch Inkonsistenzen und Unsicherheiten in den anzusetzenden Bruchkriterien sowie der initialen Gebirgsüberlagerung, respektive dem Betrag der ererbten Spannungen und den Seitendruckbeiwerten.

Im Untersuchungsgebiet können mindestens drei Generationen von Entlastungsklüften identifiziert werden, welche sich klar nach den steilstehenden, vermutlich syntektonischen S- und Q-Klüften bildeten. Die subhorizontalen, zum Teil Quarz-gefüllten L-Klüfte bildeten sich als uplift joints zuerst, da sie nicht von der lokalen Topographie beeinflusst sind und in Untertagebauwerken der externen Zentralmassive noch bei $500 \mathrm{~m}$ hoher Überlagerung auftreten (Keller et al. 1987). Es ist möglich, dass die L-Klüfte den spätalpinen Zerrklüften (Mullis 1996) und einer spät-alpinen Miocänen Hebung entsprechen.

Die auf beiden Talseiten flach bis mittelsteil talwärts einfallenden $\mathrm{T}_{1}$-Klüfte beschreiben, wenn sie über die Talsohle interpoliert werden, eine flache U-Form. Sie können als eine frühe Generation von post-uplift joints interpretiert werden, die sich parallel zu einem früheren, schwach ausgeprägten Tal entwickelt haben könnten. Eine absolute Zuordnung zu einem spezifischen Pleistozänen Erosionsstadium ist zurzeit nicht möglich. Ähnliche Beobachtungen von Entlastungsklüften, die einer früheren Topographie folgen, beschreiben Jahns (1943) sowie Chapman \& Rioux (1958) in New England (USA).

$\mathrm{T}_{2}$-Klüfte verlaufen parallel zu den heutigen lokalen Taloberflächen im Reuss- und Göschenertal und stellen die jüngsten Klüfte im Untersuchungsgebiet dar. Dies kann auch klar anhand der fraktographischen Untersuchungen bestätigt werden: Praktisch alle auf $\mathrm{T}_{2}$-Kluftflächen beobachteten Federmarken enden an den anderen Kluftsets. Der Grund liegt darin, dass sich ein Bruch nicht über freie Oberflächen ausbreiten kann (Twiss 1992). Nur vereinzelt konnten Klüfte beobachtet werden, über die Federlinien von $\mathrm{T}_{2}$-Klüfte hinweg laufen. Es ist anzunehmen, dass es sich in diesen Fällen um geschlossene, vermutlich mineralogisch verfüllte ältere Klüfte handelt, die nicht mehr als effektive Trennflächen wirksam waren. Die $\mathrm{T}_{2}$-Talklüfte bildeten sich nach dem Maximalstand der letzten Eiszeit. $\mathrm{T}_{2}$-Talklüfte erreichen Ausdehnungen von über $100 \mathrm{~m}$ und bestehen jeweils aus einer grösseren Anzahl von mit Federmarken belegten Extensionsrissen von etwa 5-10 m Grösse. Die Wachstumsrichtungen der $\mathrm{T}_{2}$-Talkklüfte sind primär subhorizontal. Ausgeprägte Federmarken können praktisch nur auf post-uplift Klüften beobachtet werden, nicht auf HebungsKlüften (uplift joints) und nur selten auf syntektonischen Klüften (Bahat 1991).

Die mittleren Abstände der $\mathrm{T}_{1}$-respektive $\mathrm{T}_{2}$-Klüfte nehmen tendenziell mit zunehmender Überlagerung zu und konnten im Vorfluterstollen des KW Göschenen bis in eine (senkrecht zur
Oberfläche gemessen) Tiefe von rund $80 \mathrm{~m}$ beobachtet werden. Diese Tiefe stimmt mit den durchgeführten felsmechanischen Berechnungen überein. Die Grösse der Talklüfte nimmt mit zunehmender Überlagerung ab, was mit der Zunahme der $\sigma_{3} / \sigma_{1}$ Hauptspannungsverhältnisse erklärt werden kann. Talklüfte bilden sich nur in granitischen Gesteinen ausserhalb einer deutlichen duktilen oder spröden alpin-tektonisch Überprägung.

$\mathrm{T}_{1}$ - und $\mathrm{T}_{2}$-Klüfte haben auch eine grosse praktische Bedeutung: $\mathrm{T}_{2}$-Klüfte unterschneiden $\mathrm{T}_{1}$-Klüfte und bilden zusammen in steilen Talflanken kinematisch instabile Bruchkörper. Da die Einfallwinkel der $\mathrm{T}_{1}$-Klüfte (>40 Grad) oft grösser als die Reibungswinkel der Kluftoberflächen sind, finden oft planare oder keilförmige Blockrutschungen statt, welche aufgrund der relativ grossen mittleren Kluftabstände im Zentralen Aaregranit zu energiereichen Blockstürzen mit Volumina von teilweise mehr als $10 \mathrm{~m}^{3}$ führen. Solche Blockstürze haben im Juni 2006 während längerer Zeit die Verkehrswege der Gotthardroute bei Gurtnellen blockiert.

$\mathrm{T}_{1}$ - und $\mathrm{T}_{2}$-Talklüfte sind aufgrund ihrer oben beschriebenen Genese räumlich ausgedehnt, persistent und zeigen oft grössere Öffnungsweiten. Dies führt zu einer sehr hohen Wasserwegsamkeit, welche sich in oberflächennahen Tunnelbauwerken durch eine intensive und rasche Gebirgsdrainage manifestiert, solange die Bauwerke unter dem hier in der Regel tief liegenden Wasserspiegel liegen (z. B. Klemenz 1974). Intensive Grundwasserneubildung an der Geländeoberfläche (z.B. durch Schneeschmelze) führt in solchen Fällen zu raschen und starken Reaktionen der Wasserzutritte auf Tunnelniveau (z.B. Keller \& Schneider 1982).

\section{Verdankungen}

Wir möchten uns bei den Reviewern P. Bossart und A.G. Milnes für ihre wertvollen und detaillierten Kommentare und Anregungen zu einer ersten Version des vorliegenden Beitrags bedanken. Weitere wichtige Diskussionsbeiträge zur Entstehung von Entlastungs- und Talklüften lieferten J. Moore, K. Leith, F. Amann (alle ETH Zürich) und M. Diederichs (Kingston CA).

\section{LITERATURVERZEICHNIS}

Bahat, D. 1991: Tectonofractography. Springer, Berlin Heidelberg.

Bahat, D., Grossenbacher, K. \& Karasaki, K. 1999: Mechanism of exfoliation joint formation in granitic rocks, Yosemite National Park. Journal of Structural Geology 21, 85-86.

Bahat, D., Rabinovitch, A. \& Frid, B. 2005: Tensile Fracturing in Rocks. Tectonofractographic and Electromagnetic Radiation Methods. Springer, Berlin, Heidelberg.

Bankwitz, P. \& Bankwitz, E. 1995: Aspekte der Entwicklung von Klüften in postkinematischen Graniten des Erzgebirges (speziell Eibenstocker Massiv). Zeitschrift für geologische Wissenschaften 23, 777-793.

Both, T. 1999: Geologie und Geotechnik im Gebiet des Etzlitals. Unpublizierte Diplomarbeit Geologisches Institut, ETH Zürich.

Bradley, W.C. 1963: Large-scale exfoliation in massive sandstones of the Colorado Plateau. Geological Society of America Bulletin 74, 519-527.

Bucher, St.P. 2006: Talklüfte im östlichen Aarmassiv (Schöllenen-Schlucht). Unpublizierte Diplomarbeit, Geologisches Institut, ETH Zürich.

Burkhard, M.1999: Strukturgeologie und Tektonik im Bereich AlpTransit. In: Löw, S. \& Wyss R. (eds.) Vorerkundung und Prognose der Basistunnels am Gotthard und am Lötschberg. Balkema, Rotterdam, 45-56. 
Challandes, N. 2001: Behavior of Rb-Sr and Ar-Ar systems in metagranite shear zones (Roffna granite and Grimsel granodiorite, Swiss Alps). PhD Dissertation, Neuchatel University.

Chapman, C.A. \& Rioux, R.L. 1958: Statistical study of topography, sheeting, and jointing in granite, Acadia National Park, Maine. American Journal of Science 256, 111-127.

Choukroune, P. \& Gapais, D. 1983: Strain pattern in the Aar Granite (Central Alps): orthogneiss developed by bulk inhomogeneous flattening. Journal of Structural Geology 5, 411-418.

Cloos, H. 1921: Der Mechanismus tiefvulkanischer Vorgänge. Friedr. Vieweg \& Sohn, Braunschweig.

Cloos, H. 1925: Einführung in die tektonische Behandlung magmatischer Erscheinungen (Granittektonik). Berlin, Bornträger.

Diederichs, M. 2003: Rock fracture and collapse under low confinement conditions. Rock Mechanics and Rock Engineering 36, 339-381.

Eberhardt, E., Stead, D., \& Stimpson, B. 1999: Quantifying pre-peak progressive fracture damage in rock during uniaxial loading. International Journal of Rock Mechanics \& Mining Sciences 36, 361-380.

Eberhardt, E., Stead, D., \& Coggan, J.S. 2004: Numerical analysis of initiation and progressive failure in natural rock slopes - the 1991 Randa rockslide. International Journal of Rock Mechancis \& Mining Sciences 41, 69-87.

Eckhardt, P.M. 1957: Zur Talgeschichte des Tavetsch, seine Bruchsysteme und jungquartären Verwerfungen. PhD Dissertation, ETH Zürich.

Florineth, D. \& Schlüchter, C. 1998: Reconstructing the Last Glacial Maximum (LGM) ice surface geometry and flowlines in the Central Swiss Alps. Eclogae Geologicae Helvetiae 91, 391-407.

Frei, B. 1999: Geologie, Hydrogeologie und Geotechnik im Gebiet der Alp Caschlè, Sedrun (GR). Unpublizierte Diplomarbeit, Geologisches Institut, ETH Zürich.

Frey, M., Bucher, K., Frank, E. and Mullis, J. 1980: Alpine metamorphism along the geotraverse Basel-Chiasso - a review. Eclogae Geologicae Helvetiae $73,527-546$

Frey, M. \& Ferreiro-Maehlmann, R. 1999: Alpine metamorphism of the Central Alps. Schweizerische Mineralogische und Petrographische Mitteilungen $79,135-154$.

Guldenfels, L. 1999: Strukturgeologie und Hydrogeologie auf der Göscheneralp. Unpublizierte Diplomarbeit Geologisches Institut, ETH Zürich.

Harland, W.B. 1957: Exfoliation joints and ice action. Journal of Glaciology $3,21,8-10$.

Hoek, E. 1968: Brittle failure of rock. In: Stagg, K. G. \& Zienkiewicz, O. C. (eds.) Rock Mechanics in Engineering Practice. Wiley, London, 99-124.

Hoek, E. and Bieniawski, Z.T. 1965: Brittle fracture propagation in rock under compression. International Journal of Fracture Mechanics 1, 137-155

Holzhausen, G.R., \& Johnson, A.M. 1979: Analyses of longitudinal splitting of uniaxially compressed rock cylinders. International Journal of Rock Mechanics \& Mining Sciences and Geomechanics Abstracts, 16; 3, 163-177.

Holzhausen, G.R. 1989: Origin of sheet structure, 1. morphology and boundary conditions. Engineering Geology 27, 225-278.

Huber, W. 1948: Petrographisch-mineralogische Untersuchungen im südöstlichen Aarmassiv. Schweizerische Mineralogische und Petrographische Mitteilungen 28, 555-642.

Jahns, R.H. 1943: Sheet structure in granites: its origin and use as a measure of glacial erosion in New England. Journal of Geology 51,71-98.

Jébrak, M. 1997: Hydrothermal breccias in vein-type ore deposits: A review of mechanisms, morphology and size distribution. Ore Geology Reviews $12,111-137$.

Keller, F. \& Schneider T.R. 1982: Der Furka-Basistunnel. Geologie und Geotechnik. Schweizer Ingenieur und Architekt 24, 512-520.

Keller, F., Wanner H. \& Schneider T.R. 1987: Geologischer Schlussbericht Gotthard-Strassentunnel. Beitrag Geologische Karte Schweiz, Geotechnische Serie 70, Schweizerische Geotechnische Kommission.

Klemenz, W. 1974: Die Hydrologie des Gebirges im Obergestelnstollen. Gas - Wasser - Abwasser 54, 287-289.

Kobel \& Partner AG 1999: AlpTransit Gotthard AG, Abschnitt Basistunnel, Teilabschnitt Amsteg, Geologie, Geotechnik, Hydrogeologie, Geologischer Bericht Bauprojekt, Dr. M. Kobel \& Partner AG, unveröffentlichter Bericht Nr. 4343-5.
Labhart, T. 1977: Aarmassiv und Gotthardmassiv. Sammlung geologischer Führer 63, Borntraeger, Berlin.

Labhart, T. 1999: Aarmassiv, Gotthardmassiv und Tavetscher Zwischenmassiv: Aufbau und Entstehungsgeschichte. In: Löw, S. \& Wyss, R. (eds.): Vorerkundung und Prognose der Basistunnels am Gotthard und am Lötschberg. A.A. Balkema, Rotterdam.

Labhart, T. 2001: Geologie der Schweiz. Ott, Thun

Laws, S. 2001: Structural, geomechanical and petrophysical properties of shear zones in the eastern Aar-massif, Switzerland. PhD Dissertation, ETH Zurich.

Liechti, H. 1933: Recherches petrographiques et tectoniques dans la vallee de Goeschenen (Canton d'Uri). Schweizerische Mineralogische und Petrographische Mitteilungen 13, 491-563.

Marquer, D. \& Gapais, D. 1985: Les massifs cristallins externes sur une transversale Guttanen-Val Bedretto (Alpes Centrales); structures et histoire cinematique. Comptes Rendus de l'Academie des Sciences, Serie 2, Mecanique, Physique, Chimie, Sciences de l'Univers, Sciences de la Terre. 301, 543-546.

Miller, D.J. \& Dunne, T. 1996: Topographic perturbations of regional stresses and consequent bedrock fracturing. Journal of Geophysical Research 101, B11, 25523-25536.

Milnes, A.G. \& Pfiffner O.A. 1980: Structural development of the Infrahelvetic Complex, eastern Switzerland. Eclogae Geologicae Helvetiae 73, 619-633.

Müller, L. 1962: Über die Entstehung oberflächenparalleler Klüfte - Versuch einer geomechanischen Erklärung. Geologie und Bauwesen 27, $146-152$.

Mullis, J. 1996: P-T-t path of quartz formation in extensional veins of the Central Alps. Schweizerische Mineralogische und Petrographische Mitteilungen 76,159-164.

Priest, S.D. 1993: Discontinuity Analysis for Rock Engineering. Chapman \& Hall, London.

Ramsay, J.G. \& Huber, M.I. 1987: The Techniques of Modern Structural Geology - Volume 2: Folds and Fractures. Academic Press, London.

Read R.S., Chandler N.A., \& Dzik E.J. 1998: In situ strength criteria for tunnel design in highly-stressed rock masses. International Journal of Rock Mechanics \& Mining Sciences 35, 261-278

RocScience 2009: Rocscience Inc. Phase2 version 7.0 - finite element analysis for excavations and slopes. www.rocscience.com

Savage, W.Z, Swolfs, H.S. \& Powers, P.S. 1985: Gravitational stresses in long symmetric ridges and valleys. International Journal of Rock Mechanics \& Mining Sciences 22, 291-302.

Segall, P. \& Pollard, D.D. 1983: Nucleation and growth of strike-slip faults in granite. Journal of Geophysical Research 88, B1, 555-568

Sibson, R. H. 1977: Fault rocks and fault mechanisms. Journal of the Geological Society London 133, 191-213.

Stalder, H. A. 1964: Petrographische und Mineralogische Untersuchungen im Grimselgebiet (Mittleres Aarmassiv). Schweizerische Mineralogische und Petrographische Mitteilungen 44, 259-267.

Steck, A. 1968: Die alpidischen Strukturen in den Zentralen Aaregraniten des westlichen Aarmassivs. Eclogae Geologicae Helvetiae 61, 19-48.

Twiss, R.J. \& Moores, E.M.1992: Structural Geology, W. H. Freeman and Company, New York.

Waltham, T. 2002: Unloading joints and rockfalls in Norway's fiordlands. Geology Today 18, 220-225.

Zangerl, C.J. 2003: Analysis of Surface Subsidence in Crystalline Rocks above the Gotthard Highway Tunnel, Switzerland. PhD Dissertation, ETH Zurich.

Zangerl, C.J, Loew, S. \& Eberhardt, E. 2006: Structure, geometry and formation of brittle discontinuities in anisotropic crystalline rocks of the central Gotthard Massif, Switzerland. Eclogae Geologicae Helvetiae 99, 271-290.

Manuscript received March 27, 2008

Revision accepted September 9, 2009

Published Online first November 30, 2009

Editorial Handling: A. G. Milnes 